\title{
Nonlinear neutrino-photon interactions inside strong laser pulses
}

\author{
Sebastian Meuren, Christoph H. Keitel and Antonino Di Piazza \\ Max-Planck-Institut für Kernphysik, \\ Saupfercheckweg 1, D-69117 Heidelberg, Germany \\ E-mail: s.meuren@mpi-hd.mpg.de, keitel@mpi-hd.mpg.de, \\ dipiazza@mpi-hd.mpg.de
}

ABSTRACT: Even though neutrinos are neutral particles and interact only via the exchange of weak gauge bosons, charged leptons and quarks can mediate a coupling to the photon field beyond tree level. Inside a relativistically strong laser field nonlinear effects in the laser amplitude can play an important role, as electrons and positrons interact nonperturbatively with the coherent part of the photon field. Here, we calculate for the first time the leadingorder contribution to the axial-vector-vector current-coupling tensor inside an arbitrary plane-wave laser field (which is taken into account exactly by employing the Furry picture). The current-coupling tensor appears in the calculation of various electroweak processes inside strong laser fields like photon emission or trident electron-positron pair production by a neutrino. Moreover, as we will see below, the axial-vector-vector current-coupling tensor contains the Adler-Bell-Jackiw (ABJ) anomaly. This occurrence renders the currentcoupling tensor also interesting from a fundamental point of view, as it is the simplest Feynman diagram in an external field featuring this kind of anomaly.

Keywords: Neutrino Physics, Electromagnetic Processes and Properties, Anomalies in Field and String Theories

ARXIV EPRINT: 1504.02722 


\section{Contents}

1 Introduction 1

2 Neutrino-photon interactions inside strong laser fields 5

2.1 Lagrangian density 6

2.2 Plane-wave background fields $\quad 7$

2.3 Z boson exchange 8

$2.4 \mathrm{~W}$ boson exchange 9

3 Current-coupling tensor $\quad 9$

$\begin{array}{lll}3.1 & \text { Ward-Takahashi identity } & 12\end{array}$

$\begin{array}{lll}3.2 & \text { Tensor structure } & 14\end{array}$

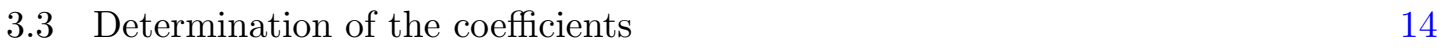

$\begin{array}{lll}3.4 & \text { Final result } & 15\end{array}$

4 Adler-Bell-Jackiw anomaly 16

$\begin{array}{llr}5 & \text { Special field configurations } & \mathbf{1 7}\end{array}$

$\begin{array}{ll}5.1 \text { Constant-crossed field } & 18\end{array}$

$\begin{array}{lll}5.2 & \text { Linear polarization } & 19\end{array}$

$\begin{array}{lll}5.3 & \text { Circular polarization } & 21\end{array}$

6 Conclusion $\quad 22$

$\begin{array}{ll}\text { A Dressed vertex } & 23\end{array}$

$\begin{array}{ll}\text { B Gamma algebra } & 24\end{array}$

$\begin{array}{ll}\text { C Summary of important relations } & 25\end{array}$

\section{Introduction}

As different neutrino mass eigenstates exist [1-7], only the lowest one is stable and all others can, in principle, decay radiatively [8-13]. However, due to the smallness of the available phase space and the Glashow-Iliopoulos-Maiani (GIM) suppression mechanism (i.e. cancellations between contributions from different fermion generations) $[13,14]$ the neutrino life time is much larger than the age of the universe (the electromagnetic properties of neutrinos in vacuum are discussed in [15-20]).

Nevertheless, a neutrino can emit photons inside strong electromagnetic background fields, which catalyze the decay. For example, strong magnetic fields encountered in various 


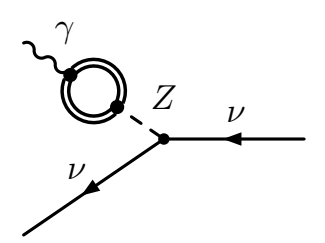

(a) Photon emission.

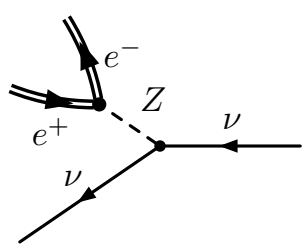

(b) Pair production.

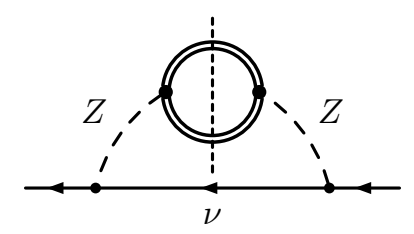

(c) Optical theorem for pair production.

Figure 1. a) Photon emission and b) trident electron-positron pair production by a neutrino inside a strong, plane-wave laser field (mediated by the neutral current, i.e. $Z$ boson exchange). For an electron neutrino also the charged current must be taken into account ( $W$ boson exchange, see figure 4). c) The total trident pair-production probability is related to the imaginary part of the neutrino self-energy diagram (see e.g. $[30,54]$ for details). The double lines denote here electron and positron states, which are dressed by the laser field (time axis from right to left).

astrophysical situations substantially reduce the neutrino life time [21-27] (see also [28], where the Coulomb field has been investigated). Inside background fields also the production of electron-positron pairs - which is not possible in vacuum due to energy-momentum conservation - is feasible under certain circumstances [29-35].

Moreover, neutrino properties like their mass and their magnetic moment are modified by electromagnetic background fields [36-38]. Implications for neutrino oscillations have been studied in [39-42] and the possibility for spin light has been pointed out in [43, 44].

The presence of electromagnetic background fields could also be exploited to create neutrinos, e.g. via photon splitting [45-47], scattering [48-50] or the trident process [51] (for a review of electroweak processes in electromagnetic background fields see $[52,53]$ ).

It is an interesting question whether the emission of photons by neutrinos or other processes like electron-positron pair production could be investigated in a laboratory experiment using high-power lasers (see figure 1). To shed light on the feasibility of this idea, the special case of a circularly polarized, monochromatic plane-wave laser field has been analyzed in [55-57] (see also [58-60]). As laser fields are naturally produced with linear polarization and the hightest intensities can only be achieved by using short laser pulses, it is desirable to generalize these results accordingly. In the present paper we will therefore consider a plane-wave laser field with arbitrary polarization and pulse shape.

Inside plane-wave laser fields the probability for a neutrino process depends primarily on the laser intensity and the neutrino energy. A convenient gauge- and Lorentz-invariant measure for the laser field strength is given by the parameter $\xi=|e| E_{0} /(m \omega c)$, where $E_{0}$ is the electric field amplitude and $\omega$ the central angular frequency of the laser $(e<0$ and $m$ denote the electron charge and mass, respectively). In the regime $\xi \gtrsim 1$ the interaction between the background field and the electron and the positron must be taken into account 
exactly by solving the Dirac equation in the presence of the background field [61-65]. For a plane-wave field this is possible analytically and one obtains the Volkov states as singleparticle states $[66,67]$. Working in momentum space, the only necessary modification of the Feynman rules is the replacement of the free vertex by the so-called dressed vertex (i.e. $-i e \gamma^{\mu} \rightarrow \Gamma^{\mu}$ for QED; see appendix A and e.g. [65, 68] for more details). Unlike in vacuum, four-momentum is conserved only up to a multiple of the laser four-momentum at the dressed vertex, which changes the kinematics of the processes.

It is well known that for $\xi \gg 1$ the formation region for single-vertex processes primed by the laser field is much smaller than the laser wavelength, such that the local constant-crossed field approximation is applicable [61, 64]. Therefore, the case of a constant-crossed background field (studied e.g. in $[30,57,69,70]$ ) is particularly interesting and provides the order of magnitude for the expected probabilities. Inside a constantcrossed field the probability depends nontrivially only on the quantum-nonlinearity parameter $\chi=\left(2 E_{\nu} / m c^{2}\right)\left(E_{0} / E_{\mathrm{cr}}\right)$, where $E_{\nu}$ denotes the energy of the incoming neutrino and $E_{\mathrm{cr}}=m^{2} c^{3} /(\hbar|e|)=1.3 \times 10^{16} \mathrm{~V} / \mathrm{cm}$ the critical field strength of QED [71-73] (the expression of $\chi$ given here assumes a head-on collision and neglects the neutrino mass).

As the nonlinear-quantum parameter is inversely proportional to the cube of the electron (positron) mass $\left(\chi \sim m^{-3}\right)$, nonlinear quantum effects caused by muon or tau leptons are strongly suppressed for reasonable parameters and ignored here. Correspondingly, the symmetry between different lepton generations is broken and the GIM mechanism does not apply. Furthermore, the laser provides additional energy and momentum to the reaction, which enlarges the available phase space. Due to these two reasons the probability for photon emission by neutrinos inside a plane-wave field is strongly enhanced in comparison with the vacuum case (note that the laser field also affects tree-level processes like the decay of a muon [74, 75]). Nevertheless, since the enhancement is primed by an electromagnetic exchange of photons between an electron/positron loop and the laser, we expect that the probabilities for nonlinear neutrino processes inside laser fields still contain the suppression factor $\left(m / M_{Z, W}\right)^{4} \sim 10^{-20}$ and an experimental observation is challenging $\left(M_{Z} \approx 91 \mathrm{GeV}\right.$ and $M_{W} \approx 80 \mathrm{GeV}$ denote the mass of the $Z$ and the $W$ boson, respectively [1]).

By combining accelerator-based neutrino beams with energies in the $\mathrm{GeV}$ range [7685] with strong optical lasers $\left(\xi \sim 10^{2-3}\right)$ [86-89], the nonlinear quantum regime $\chi \gtrsim 1$ could be entered, where also the production of real electron-positron pairs via the trident process becomes feasible [29-35]. As the energy and momentum required to bring the electron-positron pair on shell are provided by the laser field, the probability for trident pair production even exceeds the one for photon emission if $\chi \gtrsim 1$ (the corresponding Feynman diagram contains only two interaction vertices, see figure 1).

In order to calculate the probability for neutrino photon emission or trident pair production (via the optical theorem), the coupling between the vector current ( $\gamma^{\mu}$-vertex) and the axial-vector current $\left(\gamma^{5} \gamma^{\mu}\right.$-vertex $)$ described by the tensor $T_{5}^{\mu \nu}\left(q_{1}, q_{2}\right)$ must be determined (see figure 2). For a constant background field it has been investigated in [95-102]. In the present paper an arbitrary plane-wave laser field is considered as background field (see section 2.2) and a triple-integral representation for $T_{5}^{\mu \nu}\left(q_{1}, q_{2}\right)$ is derived, which can be transformed into a double-integral representation using the relations given in [54]. Special 


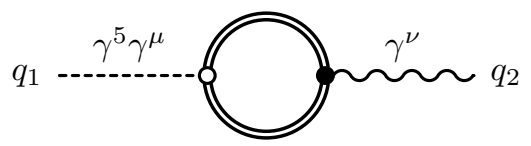

(a) Current-coupling tensor $T_{5}^{\mu \nu}\left(q_{1}, q_{2}\right)$.

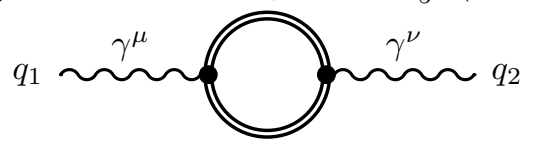

(b) Polarization tensor $T^{\mu \nu}\left(q_{1}, q_{2}\right)$.

Figure 2. a) The leading-order Feynman diagram for the coupling between the vector current $\left(\gamma^{\mu}\right.$-vertex) and the axial-vector current $\left(\gamma^{5} \gamma^{\mu}\right.$-vertex $)$, see eq. (2.13). b) The current-coupling tensor $T_{5}^{\mu \nu}\left(q_{1}, q_{2}\right)$ is closely related to the polarization tensor $T^{\mu \nu}\left(q_{1}, q_{2}\right)$, which was considered e.g. in [68, 90-94], see eq. (2.12). Solid lines indicate fermions, double lines Volkov states (which take the plane-wave background field exactly into account), wiggly lines photons and dashed lines the axial-vector current.

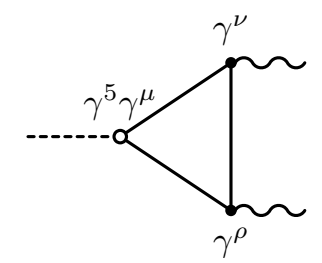

(a) Anomalous triangle diagram.

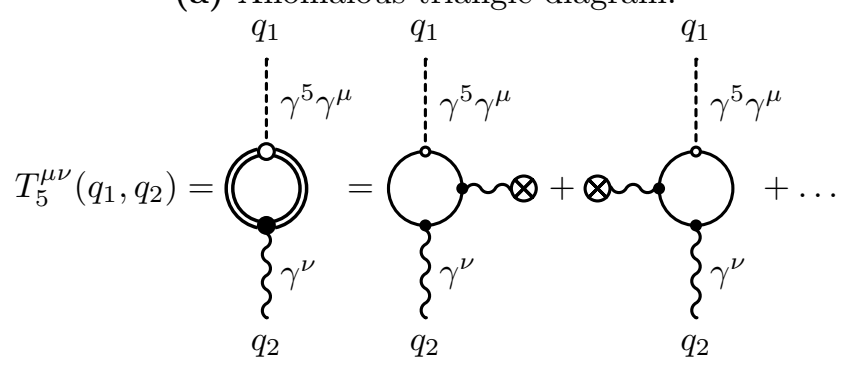

(b) Leading-order expansion in the external field.

Figure 3. a) The axial-vector anomaly in vacuum QED is caused by the triangle diagram. b) As for weak external fields $A^{\mu}(\phi)$ (denoted by $\otimes$ ) the leading-order field-dependent contribution to the current-coupling tensor $T_{5}^{\mu \nu}\left(q_{1}, q_{2}\right)$ [see eq. (2.13)] corresponds to the triangle diagram, one also expects an anomalous term in the Ward-Takahashi identity for $T_{5}^{\mu \nu}\left(q_{1}, q_{2}\right)$ [see eq. (3.25)]. Here, solid lines indicate the vacuum states and double lines dressed Volkov states for the charged fermions, wiggly lines photons and dashed lines the axial-vector current.

attention is payed to the Ward-Takahashi identity, which contains a contribution due to the Adler-Bell-Jackiw (ABJ) anomaly (see figure 3) [103, 104]. The anomalous term is calculated explicitly by applying a suitable regularization procedure.

The present paper is organized as follows: in section 2 the interaction between neutrinos and photons inside a plane-wave background field is considered and it is shown how the axial-vector-vector current-coupling tensor $T_{5}^{\mu \nu}\left(q_{1}, q_{2}\right)$ (see figure 2) appears naturally in the electroweak sector of the standard model if plane-wave background fields are taken into 


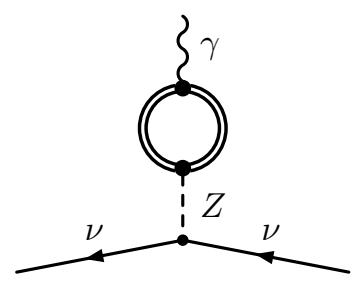

(a) $Z$ boson exchange.

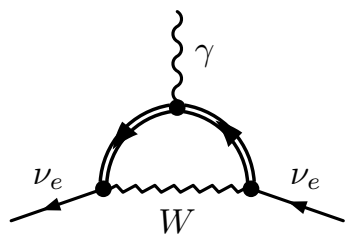

(b) $W$ boson exchange.

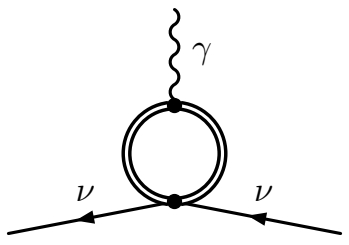

(c) Local limit (Fermi description).

Figure 4. Neutrino-photon interaction vertex. a) The electron-positron loop interacts via the neutral current with all neutrino flavor states. b) Electron neutrinos also couple via the charged current to electrons and positrons. c) In the local limit (exchanged momentum much smaller than the weak gauge-boson mass) the effective four-point Fermi interaction is obtained. The double lines denote dressed electron and positron states which take into account exactly the laser field (time axis from right to left).

account. The calculation of $T_{5}^{\mu \nu}\left(q_{1}, q_{2}\right)$ is then presented in section 3 , followed by a detailed discussion of the ABJ anomaly in section 4. Subsequently, various important special cases like a constant-crossed and a circularly polarized, monochromatic field are considered in section 5 and compared with known expressions from the literature. Finally, summary and conclusions are provided in section 6 .

From now on we use natural units $\hbar=c=1$ and Heaviside-Lorentz units for charge $\left[\alpha=e^{2} /(4 \pi) \approx 1 / 137\right.$ denotes the fine-structure constant], the notation agrees with [68].

\section{Neutrino-photon interactions inside strong laser fields}

As neutrinos are neutral particles, their interaction with photons must be mediated by loop diagrams which contain electrically charged particles (see figure 4). At the loop level the quantization of the electroweak sector of the standard model involves "unphysical" degrees of freedom, i.e. particles which appear only in loops but not as free, asymptotic states $[105,106]$. These are the unphysical scalar Higgs particles, present if the calculation is performed in a renormalizable gauge (from the Higgs doublet, which consists of four scalar fields, only one degree of freedom corresponds to the physically observable Higgs particle) and the Feynman-Faddeev-Popov ghosts, which appear in the quantization of a nonabelian gauge theory $[107,108]$. Therefore, the complete set of Feynman rules for the electroweak sector of the standard model after symmetry breaking is rather large [109-112]. Fortunately, the leading-order contribution (with respect to the electroweak mass scale) to the neutrino-photon coupling inside a plane-wave background field is given by only two diagrams, which are shown in figure 4 (see also [17, 57]). 
Due to the existence of neutrino oscillations we know that neutrinos have a finite mass $[1-7,113]$. The left-handed neutrino mass eigenstates $\nu_{r L}(r=1,2,3)$ are related by the unitary Pontecorvo-Maki-Nakagawa-Sakata (PMNS) matrix $\mathbf{U}_{\alpha r}$ (which is also simply called neutrino mixing matrix) $\nu_{\alpha L}=\mathbf{U}_{\alpha r} \nu_{r L}[1,114-116]$ to the left-handed flavor neutrino eigenstates $\nu_{\alpha L}(\alpha=e, \mu, \tau)$. As neutrinos are produced via the charged current as left-handed flavor eigenstates, the nature of their right-handed component (required for the construction of a mass term in the Lagrangian) is not determined so far, i.e. the neutrino could be either a Dirac or a Majorana particle. At high energies, however, the neutrino mass can usually be neglected and with a reasonable experimental precision it is not possible to distinguish between Dirac and Majorana neutrinos. Correspondingly, we can assume in the following that the neutrino is a massless, left-handed Dirac particle as originally postulated in the standard model.

\section{$2.1 \quad$ Lagrangian density}

After electroweak-symmetry breaking the Lagrangian density, which describes the interaction between the photon field $\mathcal{A}^{\mu}$ and the various fermion fields $\psi_{f}$, is given by $[1,106]$

$$
\mathcal{L}_{\mathrm{L}}^{\mathrm{EM}}=e \mathcal{A}_{\mu} J_{\mathrm{EM}}^{\mu}, \quad J_{\mathrm{EM}}^{\mu}=-\bar{\psi}_{e} \gamma^{\mu} \psi_{e}+\frac{2}{3} \bar{\psi}_{u} \gamma^{\mu} \psi_{u}-\frac{1}{3} \bar{\psi}_{d} \gamma^{\mu} \psi_{d}+\ldots
$$

[the index $f=e, \mu, \tau, \ldots$ labels the type of fermion field (quarks and leptons), Dirac spinor indices are suppressed (note that we use the convention $e<0$ )]. Correspondingly, we obtain

$$
\mathcal{L}_{\mathrm{L}}^{\mathrm{Z}}=-\frac{g}{2 \cos \theta_{W}} Z_{\mu} J_{\mathrm{Z}}^{\mu}, \quad J_{\mathrm{Z}}^{\mu}=\bar{\psi}_{f}\left[g_{v}^{(f)} \gamma^{\mu}+g_{a}^{(f)} \gamma^{\mu} \gamma^{5}\right] \psi_{f}
$$

for the interaction with the $Z$ boson field $Z^{\mu}$. Here, $g=g_{2}$ and $g^{\prime}=g_{1}=g \tan \theta_{W}$ are the fundamental coupling constants for weak isospin and hypercharge, respectively, which are (after the symmetry breaking) related to the electron charge $e$ and the Fermi constant $G_{F}$ by $-e=g \sin \theta_{W}$ and $G_{F}=\left(g^{2} \sqrt{2}\right) /\left(8 M_{W}^{2}\right) \approx 1.17 \times 10^{-5} \mathrm{GeV}^{-2}$, respectively (at tree level the gauge-boson masses obey $M_{W}=M_{Z} \cos \theta_{W}, \theta_{W}$ is called the weak mixing or Weinberg angle). The constants $g_{v}^{(f)}$ and $g_{a}^{(f)}$ depend on the type of fermion. For the charged leptons we obtain $g_{v}^{(e, \mu, \tau)}=-1 / 2+2 \sin ^{2} \theta_{W}$ and $g_{a}^{(e, \mu, \tau)}=-1 / 2$, for the (massless) neutrinos $g_{v}^{\left(\nu_{e}, \nu_{\mu}, \nu_{\tau}\right)}=g_{a}^{\left(\nu_{e}, \nu_{\mu}, \nu_{\tau}\right)}=1 / 2$ and for the quarks $g_{v}^{(u, c, t)}=1 / 2-(4 / 3) \sin ^{2} \theta_{W}$ $g_{v}^{(d, s, b)}=-1 / 2+(2 / 3) \sin ^{2} \theta_{W}, g_{a}^{(u, c, t)}=-g_{a}^{(d, s, b)}=1 / 2$ [note that we use the same notation for $\gamma^{5}$ as in [67] and [106], i.e. the projection operators $P_{L}$ for the left-handed and $P_{R}$ for the right-handed component are given by $P_{L}=\left(\mathbf{1}+\gamma^{5}\right) / 2$ and $P_{R}=\left(\mathbf{1}-\gamma^{5}\right) / 2$, respectively].

Finally, the Lagrangian density, which describes the interaction between the complex $W$ boson field $W_{\mu}^{+}$[the plus is part of the symbol name, we also define $\left.W_{\mu}^{-}=\left(W_{\mu}^{+}\right)^{\dagger}\right]$ and the first lepton generation can be written as

$$
\mathcal{L}_{e}^{\mathrm{W}}=-\frac{g}{2 \sqrt{2}}\left[W_{\mu}^{+} J_{\mathrm{W}, e}^{\mu}+W_{\mu}^{-}\left(J_{\mathrm{W}, e}^{\mu}\right)^{\dagger}\right], \quad J_{\mathrm{W}, e}^{\mu}=\bar{\psi}_{\nu_{e}} \gamma^{\mu}\left(\mathbf{1}+\gamma^{5}\right) \psi_{e} .
$$

From eqs. (2.1)-(2.3) one obtains the interaction vertices between the fermions and the electroweak gauge fields of the standard model [106, 109]; they contain both vector $\left(\gamma^{\mu}\right)$ and axial-vector $\left(\gamma^{\mu} \gamma^{5}\right)$ couplings. 
After quantization the propagators for the weak gauge bosons are (in position space and Feynman gauge) given by [106]

$$
i G_{Z, W}^{\mu \nu}(x-y)=\int \frac{d^{4} p}{(2 \pi)^{4}} \frac{-i g^{\mu \nu}}{p^{2}-M_{Z, W}^{2}+i 0} e^{-i p(x-y)} .
$$

If the exchanged momenta are much smaller than the weak mass scale $M_{Z, W} \sim 100 \mathrm{GeV}$, one can neglect the momentum dependence in the denominator in eq. (2.4) (local limit). After taking the momentum integrals, the propagators are then given by

$$
i G_{Z, W}^{\mu \nu}(x-y)=i \frac{g^{\mu \nu}}{M_{Z, W}^{2}} \delta^{4}(x-y)
$$

(see figure 4c). Physically, this means that the $Z$ and the $W$ boson are too heavy to propagate a significant distance and we obtain essentially Fermi's description for the weak force $[117,118]$.

\subsection{Plane-wave background fields}

In the following we will consider an external plane-wave laser field described by the field tensor

$$
F^{\mu \nu}(\phi)=\partial^{\mu} A^{\nu}(\phi)-\partial^{\nu} A^{\mu}(\phi)=\sum_{i=1,2} f_{i}^{\mu \nu} \psi_{i}^{\prime}(\phi)
$$

$\phi=k x$ (the prime denotes the derivative with respect to the argument). Here, $A^{\mu}(\phi)$ denotes the four-potential and

$$
f_{i}^{\mu \nu}=k^{\mu} a_{i}^{\nu}-k^{\nu} a_{i}^{\mu}, \quad f_{i \rho}^{\mu} f_{j}^{\rho \nu}=-\delta_{i j} a_{i}^{2} k^{\mu} k^{\nu}, \quad k_{\mu} f_{i}^{\mu \nu}=0
$$

$\left(k^{2}=k a_{i}=a_{1} a_{2}=0\right)$. We also introduce the integrated field tensor

$$
\mathfrak{F}^{\mu \nu}(\phi)=\int_{-\infty}^{\phi} d \phi^{\prime} F^{\mu \nu}\left(\phi^{\prime}\right)=\sum_{i=1,2} f_{i}^{\mu \nu} \psi_{i}(\phi) .
$$

Correspondingly, the scalar functions $\psi_{i}(\phi)$ describe the shape of the laser field. They are arbitrary (differentiable) functions, restricted only by the physical requirement that the external field is of finite extent and has no dc component [i.e., $\psi_{i}( \pm \infty)=\psi_{i}^{\prime}( \pm \infty)=0$, with $\psi_{i}(\phi), \psi_{i}^{\prime}(\phi)$ vanishing fast enough at infinity]. Furthermore, we assume (without restriction) that $\left|\psi_{i}(\phi)\right|,\left|\psi_{i}^{\prime}(\phi)\right| \lesssim 1$. This implies that the field strength is measured by the following (classical) intensity parameters

$$
\xi_{i}=\frac{|e|}{m} \sqrt{-a_{i}^{2}}
$$

Calculations with plane-wave background fields become particularly transparent if lightcone coordinates are used [65, 119, 120]

$$
v^{-}=v k, \quad v^{+}=v \bar{k}, \quad v^{\mathrm{I}}=v e_{1}, \quad v^{\mathrm{II}}=v e_{2}
$$

( $v^{\mu}$ is an arbitrary four-vector, I and II are also summarized as $\perp$ ). Here, we require that the four four-vectors $k^{\mu}, \bar{k}^{\mu}, e_{1}^{\mu}$ and $e_{2}^{\mu}$ form a light-cone basis [see appendix C and eq. (32) of ref. [68] and ref. [121]].

More details can be found in refs. [62-65] and in the recent review articles [61, 122-125]. 


\section{$2.3 \quad \mathrm{Z}$ boson exchange}

In the local limit the matrix element for the emission of a photon with four-momentum $q^{\mu}$ and polarization four-vector $\epsilon^{\mu}$ by a neutrino due to $Z$ boson exchange (see figure 4a) is given by [25]

$$
i \mathfrak{M}_{Z}\left(p^{\prime}, q ; p\right)=\frac{2 G_{F}}{\sqrt{2}} \bar{u}_{\nu, p^{\prime}} \gamma_{\mu} P_{L} u_{\nu, p} \frac{1}{e}\left[g_{v}^{(e)} T^{\mu \nu}\left(p-p^{\prime}, q\right)+g_{a}^{(e)} T_{5}^{\mu \nu}\left(p-p^{\prime}, q\right)\right] \epsilon_{\nu}^{*}
$$

(we use the same conventions for matrix elements as in [54]). Here $u_{\nu, p}$ and $u_{\nu, p^{\prime}}$ are the Dirac spinors for the incoming neutrino with four-momentum $p^{\mu}$ and the outgoing neutrino with four-momentum $p^{\prime \mu}$, respectively. Furthermore, $T^{\mu \nu}\left(q_{1}, q_{2}\right)$ denotes the polarization tensor (see figure 2 and [68, 90-94])

$$
T^{\mu \nu}\left(q_{1}, q_{2}\right)=\int \frac{d^{4} p d^{4} p^{\prime}}{(2 \pi)^{8}} \operatorname{tr} \Gamma^{\mu}\left(p^{\prime}, q_{1}, p\right) \frac{\not p+m}{p^{2}-m^{2}+i 0} \Gamma^{\nu}\left(p,-q_{2}, p^{\prime}\right) \frac{\not p^{\prime}+m}{p^{\prime 2}-m^{2}+i 0}
$$

and $T_{5}^{\mu \nu}\left(q_{1}, q_{2}\right)$ the axial-vector-vector current-coupling tensor

$$
T_{5}^{\mu \nu}\left(q_{1}, q_{2}\right)=\int \frac{d^{4} p d^{4} p^{\prime}}{(2 \pi)^{8}} \operatorname{tr} \Gamma^{\mu}\left(p^{\prime}, q_{1}, p\right) \gamma^{5} \frac{\not p+m}{p^{2}-m^{2}+i 0} \Gamma^{\nu}\left(p,-q_{2}, p^{\prime}\right) \frac{\not p^{\prime}+m}{p^{2}-m^{2}+i 0},
$$

which will be calculated in section 3 [the final result is given in eq. (3.38); for the definition of the dressed vertex $\Gamma^{\mu}$ see appendix A]. Note that $T_{5}^{\mu \nu}\left(q_{1}, q_{2}\right)$ is actually a pseudo-tensor and that in our definition the electron charge $e$ is taken as the coupling constant for both vertices [this is the reason for the prefactor 1/e appearing in eq. (2.11)]. Furthermore, the appearance of $g_{v}^{(e)}$ and $g_{a}^{(e)}$ in eq. (2.11) is related to the electron-positron loop and is independent of the neutrino species which interacts with the photon.

Despite the fact that the electron and the positron are the lightest charged fermions, also the muon, the tau and the various quarks contribute to the loop which couples the photon with the $Z$ boson (see figure 4a). To calculate the contribution of the other charged fermions to this loop, the electron (positron) mass and charge appearing in $\frac{1}{e} T^{\mu \nu}\left(q_{1}, q_{2}\right)$ and $\frac{1}{e} T_{5}^{\mu \nu}\left(q_{1}, q_{2}\right)$ must be replaced accordingly and the $Z$ boson coupling constants $g_{v, a}^{(e)} \rightarrow g_{v, a}^{(f)}$ adjusted. As discussed in the introduction, the nonlinear interaction with the background field can be neglected for fermions with a mass well above the electron (positron) scale (for reasonable field strengths of the background field). However, the contribution of all fermions in each generation is needed for the cancellation of the axial-vector anomaly. As the anomalous contribution to $\frac{1}{e} T_{5}^{\mu \nu}\left(q_{1}, q_{2}\right)$ is independent of the fermion mass and proportional to the square of the electric charge [at one loop in the presence of a planewave, see eq. (3.35)], this cancellation (for each fermion generation) can be seen from the relation

$$
\frac{1}{2}\left[-(-1)^{2}+3(2 / 3)^{2}-3(-1 / 3)^{2}\right]=0
$$

(all gauge-symmetry anomalies must cancel in the standard model, otherwise it would be nonrenormalizable [126]). 


\subsection{W boson exchange}

For electron neutrinos also the exchange of a $W$ boson contributes to the photon-emission matrix element (see figure $4 \mathrm{~b}$ ). Applying the local limit for the $W$ boson propagator, we obtain the following expression for the matrix element

$$
i \mathfrak{M}_{W}\left(p^{\prime}, q ; p\right)=\frac{4 G_{F}}{\sqrt{2}} \int \frac{d p_{1}^{4} d p_{2}^{4}}{(2 \pi)^{8}} \int d^{4} x e^{-i\left(p-p^{\prime}\right) x} \bar{u}_{\nu, p^{\prime}} \gamma^{\rho} P_{L} M^{\nu}\left(p_{1}, p_{2}, q ; x\right) \epsilon_{\nu}^{*} \gamma_{\rho} P_{L} u_{\nu, p},
$$

where

$$
M^{\nu}\left(p_{1}, p_{2}, q ; x\right)=i E_{p_{1}, x} \frac{\not p_{1}+m}{p_{1}^{2}-m^{2}+i 0} \Gamma^{\nu}\left(p_{1},-q, p_{2}\right) \frac{\not p_{2}+m}{p_{2}^{2}-m^{2}+i 0} \bar{E}_{p_{2}, x} .
$$

Using the identities given in appendix B for an arbitrary $4 \times 4$ matrix $\Gamma$ in spinor space, we obtain

$$
\gamma^{\rho} P_{L} \Gamma \gamma_{\rho} P_{L}=-2 r_{\mu} \gamma^{\mu} P_{L}
$$

where

$$
r_{\mu}=\frac{1}{2} \operatorname{tr} P_{R} \gamma_{\mu} \Gamma=\frac{1}{2} \operatorname{tr} \gamma_{\mu} P_{L} \Gamma .
$$

Therefore, we can write the matrix element for the $W$ boson exchange diagram as [see eq. (2.15)] [25]

$$
i \mathfrak{M}_{W}\left(p^{\prime}, q ; p\right)=\frac{2 G_{F}}{\sqrt{2}} \bar{u}_{\nu, p^{\prime}} \gamma_{\mu} P_{L} u_{\nu, p} \frac{1}{e}\left[T^{\mu \nu}\left(p-p^{\prime}, q\right)+T_{5}^{\mu \nu}\left(p-p^{\prime}, q\right)\right] \epsilon_{\nu}^{*}
$$

(note that it only contributes for electron neutrinos). In the local limit it has the same structure as the one for the $Z$ boson exchange given in eq. (2.11). The anomaly, however, must drop also for this diagram if one first performs the calculations by employing the full $W$ boson propagator in eq. (2.4) [25].

\section{Current-coupling tensor}

In the previous section it was shown how the axial-vector-vector current-coupling tensor $T_{5}^{\mu \nu}\left(q_{1}, q_{2}\right)$ (see figure 2) arises in the calculation of neutrino-photon interactions inside strong laser fields. Now, we will examine $T_{5}^{\mu \nu}\left(q_{1}, q_{2}\right)$ closer and derive a compact tripleintegral representation for it. After applying the Feynman rules [61-65], we obtain the following expression [see eq. (2.13)]

$$
T_{5}^{\mu \nu}\left(q_{1}, q_{2}\right)=\int \frac{d^{4} p d^{4} p^{\prime}}{(2 \pi)^{8}} \operatorname{tr} \Gamma^{\mu}\left(p^{\prime}, q_{1}, p\right) \gamma^{5} \frac{\not p+m}{p^{2}-m^{2}+i 0} \Gamma^{\nu}\left(p,-q_{2}, p^{\prime}\right) \frac{\not p^{\prime}+m}{p^{\prime 2}-m^{2}+i 0}
$$

(for the definition of the dressed vertex $\Gamma^{\rho}$ see appendix A). Note that $T_{5}^{\mu \nu}\left(q_{1}, q_{2}\right)$ is equivalent to the tensor

$$
\widetilde{T}_{5}^{\mu \nu}\left(q_{1}, q_{2}\right)=\int \frac{d^{4} p d^{4} p^{\prime}}{(2 \pi)^{8}} \operatorname{tr} \Gamma^{\mu}\left(p^{\prime}, q_{1}, p\right) \frac{\not p+m}{p^{2}-m^{2}+i 0} \Gamma^{\nu}\left(p,-q_{2}, p^{\prime}\right) \gamma^{5} \frac{\not p^{\prime}+m}{p^{\prime 2}-m^{2}+i 0},
$$

which is obtained by interchanging the vector- and the axial-vector current vertex $\left[\widetilde{T}_{5}^{\mu \nu}\left(q_{1}, q_{2}\right)=T_{5}^{\nu \mu}\left(-q_{2},-q_{1}\right)\right]$. 
The current-coupling tensor $T_{5}^{\mu \nu}\left(q_{1}, q_{2}\right)$, which we consider here, differs from the polarization tensor $T^{\mu \nu}\left(q_{1}, q_{2}\right)$ [see eq. (2.12)] only by the insertion of $\gamma^{5}$ (i.e. by the trace). Hence, the calculation of $T_{5}^{\mu \nu}\left(q_{1}, q_{2}\right)$ is related to the one of $T^{\mu \nu}\left(q_{1}, q_{2}\right)$ carried out in [68] and we will focus here mainly on the differences between both derivations. At first sight one may think that the small modification of the trace should only affect the technical details of the calculation. However, it is responsible for several important qualitative changes like the appearance of the ABJ anomaly in $T_{5}^{\mu \nu}\left(q_{1}, q_{2}\right)$, which we will discuss now in detail.

An important consequence of the additional $\gamma^{5}$ in the trace of $T_{5}^{\mu \nu}\left(q_{1}, q_{2}\right)$ is the fact that only an odd number of couplings to the background field are allowed if the background field is treated perturbatively (this follows from a generalization of Furry's theorem, see e.g. [127]; for the polarization tensor only an even number of couplings is possible). Accordingly, the tensor structure of $T_{5}^{\mu \nu}\left(q_{1}, q_{2}\right)$ is different from that of $T^{\mu \nu}\left(q_{1}, q_{2}\right)$ [see eq. (3.34)] and the vacuum contribution to $T_{5}^{\mu \nu}\left(q_{1}, q_{2}\right)$ vanishes (see figure 3 ). Furthermore, we will see that no infinities are encountered and $T_{5}^{\mu \nu}\left(q_{1}, q_{2}\right)$ does not need to be regularized.

In order to determine $T_{5}^{\mu \nu}\left(q_{1}, q_{2}\right)$ we insert the dressed vertex (see appendix A) into eq. (2.13) [we will denote the vertex integrals associated with $\Gamma^{\mu}\left(p^{\prime}, q_{1}, p\right)$ and $\Gamma^{\nu}\left(p,-q_{2}, p^{\prime}\right)$ by $d^{4} x$ and $d^{4} y$, respectively] and obtain

$$
T_{5}^{\mu \nu}\left(q_{1}, q_{2}\right)=4(-i e)^{2} \int \frac{d^{4} p d^{4} p^{\prime}}{(2 \pi)^{8}} \int d^{4} x d^{4} y \frac{\frac{1}{4} \operatorname{tr}[\cdots]_{5}^{\mu \nu}}{\left(p^{2}-m^{2}+i 0\right)\left(p^{\prime 2}-m^{2}+i 0\right)} e^{i S_{T}},
$$

where

$$
\begin{aligned}
i S_{T}= & i\left(p^{\prime}-p-q_{1}\right) x+i\left(p-p^{\prime}+q_{2}\right) y \\
& +i \int_{k y}^{k x} d \phi^{\prime}\left[\frac{e p_{\mu} p_{\nu}^{\prime} \mathfrak{F}^{\mu \nu}\left(\phi^{\prime}\right)}{(k p)\left(k p^{\prime}\right)}+\frac{e^{2}\left(k p-k p^{\prime}\right)}{2(k p)^{2}\left(k p^{\prime}\right)^{2}} p_{\mu} p_{\nu}^{\prime} \mathfrak{F}^{2 \mu \nu}\left(\phi^{\prime}\right)\right]
\end{aligned}
$$

and the trace in eq. (3.3) is given by

$$
\begin{aligned}
\frac{1}{4} \operatorname{tr}[\cdots]_{5}^{\mu \nu}= & \frac{1}{4} \operatorname{tr}\left[\gamma_{\alpha} a^{\alpha \mu}+i \gamma_{\alpha} \gamma^{5} b^{\alpha \mu}\right] \gamma^{5}(\not p+m)\left[\gamma_{\beta} c^{\beta \nu}+i \gamma_{\beta} \gamma^{5} d^{\beta \nu}\right]\left(\not p^{\prime}+m\right) \\
= & i m^{2}\left[\left(b^{\alpha \mu} c_{\alpha}{ }^{\nu}\right)-\left(a^{\alpha \mu} d_{\alpha}{ }^{\nu}\right)\right]-i\left(p p^{\prime}\right)\left[\left(a^{\alpha \mu} d_{\alpha}{ }^{\nu}\right)+\left(b^{\alpha \mu} c_{\alpha}{ }^{\nu}\right)\right] \\
& +i\left(p_{\alpha} p_{\beta}^{\prime}+p_{\beta} p_{\alpha}^{\prime}\right)\left(b^{\alpha \mu} c^{\beta \nu}+a^{\alpha \mu} d^{\beta \nu}\right)-i \epsilon_{\rho \sigma \alpha \beta} p^{\rho} p^{\sigma \sigma}\left(b^{\alpha \mu} d^{\beta \nu}-a^{\alpha \mu} c^{\beta \nu}\right),
\end{aligned}
$$

where

$$
\begin{aligned}
a^{\alpha \mu}=G^{\alpha \mu}\left(k p^{\prime}, k p ; k x\right), & c^{\beta \nu}=G^{\beta \nu}\left(k p, k p^{\prime} ; k y\right), \\
b^{\alpha \mu}=G_{5}^{\alpha \mu}\left(k p^{\prime}, k p ; k x\right), & d^{\beta \nu}=G_{5}^{\beta \nu}\left(k p, k p^{\prime} ; k y\right)
\end{aligned}
$$

[the additional $\gamma^{5}$ in eq. (3.5) exchanges the vector and the axial-vector current in comparison with the polarization tensor].

Next, we employ the proper time parametrization for the propagators

$$
\begin{aligned}
\frac{1}{p^{2}-m^{2}+i 0} \frac{1}{p^{2}-m^{2}+i 0}= & (-i)^{2} \int_{0}^{\infty} d s d t \\
& \times \exp \left[i\left(p^{2}-m^{2}+i 0\right) s+i\left(p^{2}-m^{2}+i 0\right) t\right]
\end{aligned}
$$

(the pole prescription $i 0$ will be dropped in the exponents and can be restored by substituting $\left.m^{2} \rightarrow m^{2}-i 0\right)$. In the final representation we change the proper-time integrals in 
the following way

$$
\int_{0}^{\infty} d s d t f(s, t)=\frac{1}{2} \int_{-1}^{+1} d v \int_{0}^{\infty} d \tau \tau \tilde{f}(\tau, v)
$$

(note that terms odd in $v$ in the resulting function $\tilde{f}(\tau, v)$ can be dropped), where

$$
\tau=s+t, \quad v=\frac{s-t}{s+t}, \quad \mu=\frac{s t}{s+t}=\frac{1}{4} \tau\left(1-v^{2}\right) .
$$

After including the parametrization (3.7) in eq. (3.3) and by adding the source terms $i p_{\mu} j^{\mu}+i p_{\mu}^{\prime} j^{\prime \mu}$ to the resulting phase in the same equation, we apply the replacements

$$
p^{\mu} \longrightarrow(-i) \partial_{j}^{\mu}, \quad p^{\prime \mu} \longrightarrow(-i) \partial_{j^{\prime}}^{\mu}
$$

to the trace given in eq. (3.5), use modified light-cone coordinates [see eq. (C11) in [68]] and define

$$
\begin{aligned}
\lambda^{\mu} & =-\frac{m(k q)}{(k p)\left(k p^{\prime}\right)} \sum_{i=1,2} \xi_{i} \Lambda_{i}^{\mu} \int_{k y}^{k x} d \phi^{\prime} \psi_{i}\left(\phi^{\prime}\right), \\
\Lambda & =-\frac{m^{2}(k q)}{2(k p)\left(k p^{\prime}\right)} \sum_{i=1,2} \xi_{i}^{2} \int_{k y}^{k x} d \phi^{\prime} \psi_{i}^{2}\left(\phi^{\prime}\right), \\
I_{i} & =-\frac{1}{2 k q \mu} \int_{k y}^{k x} d \phi^{\prime} \psi_{i}\left(\phi^{\prime}\right), \quad J_{i}=-\frac{1}{2 k q \mu} \int_{k y}^{k x} d \phi^{\prime} \psi_{i}^{2}\left(\phi^{\prime}\right)
\end{aligned}
$$

(see appendix $\mathrm{C}$ for more details). Finally, we obtain the following structure for the currentcoupling tensor

$$
T_{5}^{\mu \nu}\left(q_{1}, q_{2}\right)=4(-i e)^{2} \int \frac{d^{4} p d^{4} p^{\prime}}{(2 \pi)^{8}} \int d^{4} x d^{4} y(-i)^{2} \int_{0}^{\infty} d s d t \frac{1}{4} \operatorname{tr}[\cdots]_{5}^{\mu \nu} e^{i S_{T}^{\prime}}
$$

where the full phase including the proper-time exponents and the source terms is given by

$$
S_{T}^{\prime}=\tilde{S}_{T}+p_{\mu} j^{\mu}+p_{\mu}^{\prime} j^{\prime \mu}, \quad \tilde{S}_{T}=\left(p^{2}-m^{2}\right) s+\left(p^{\prime 2}-m^{2}\right) t+S_{T}
$$

(if no explicit argument is present, the prime is a part of the symbol name and does not indicate a derivative). After taking most of the integrals, we obtain the result [see eq. (64) in [68]]

$$
\begin{aligned}
T_{5}^{\mu \nu}\left(q_{1}, q_{2}\right)= & -2 i \pi e^{2} \delta^{(-, \perp)}\left(q_{1}-q_{2}\right) \int_{0}^{\infty} d s d t \\
& \times\left.\int_{-\infty}^{+\infty} d x^{-} \frac{1}{(s+t)^{2}} \frac{1}{4} \operatorname{tr}[\cdots]_{5}^{\mu \nu} e^{i S_{T}^{\prime}}\right|_{j=j^{\prime}=0},
\end{aligned}
$$

where $x^{-}=k x=\phi$ and the trace is given in eq. (3.5) with the replacement in eq. (3.10) and

$$
\begin{aligned}
& i S_{T}^{\prime}=i\left[\left(q_{2}^{+}-q_{1}^{+}\right) x^{-}-m^{2}(s+t)+\frac{s t}{s+t} q_{2}^{2}\right. \\
&-\frac{1}{s+t}\left(t q_{2} j-s q_{2} j^{\prime}\right)-\frac{1}{4(s+t)}\left(j+j^{\prime}\right)^{2} \\
&\left.\quad-\frac{1}{2(s+t)}\left(j+j^{\prime}\right) \lambda-\frac{1}{4(s+t)} \lambda^{2}+\Lambda\right]
\end{aligned}
$$


[note that, since no confusion can arise, we use the same symbol for the phase before and after the mentioned integrals are taken, see eqs. (3.13) and (3.14) and eqs. (3.15) and (3.16)].

Due to the momentum-conserving delta functions in eq. (3.15) we will simply write $q^{\mu}$ whenever $q_{1}^{\mu}$ and $q_{2}^{\mu}$ can be used interchangeably.

To obtain a symmetric expression with respect to the external momenta $q_{1}^{\mu}$ and $q_{2}^{\mu}$, we will perform below the shift

$$
z^{-}=x^{-}+\mu q^{-}
$$

and use $z^{-}$as integration variable.

\subsection{Ward-Takahashi identity}

According to Noether's theorem, the gauge invariance of the QED Lagrangian implies electric charge conservation. More specifically, if the spinor field $\psi(x)$ obeys the Dirac equation

$$
[i \not \partial-e \not(x)-m] \psi(x)=0
$$

[here $A^{\mu}(x)$ denotes the classical background field], the vector current is conserved

$$
\partial_{\mu} j^{\mu}(x)=0, \quad j^{\mu}(x)=\bar{\psi}(x) \gamma^{\mu} \psi(x) .
$$

After changing to momentum space

$$
j_{q}^{\mu}=\int d^{4} x e^{-i q x} j^{\mu}(x)
$$

the current conservation law is expressed by

$$
\int d^{4} x e^{-i q x}\left[\partial_{\mu} j^{\mu}(x)\right]=i q_{\mu} j_{q}^{\mu}=0 .
$$

Therefore, one expects that the polarization tensor $T^{\mu \nu}\left(q_{1}, q_{2}\right)$ obeys the following homogeneous Ward-Takahashi identity

$$
q_{1 \mu} T^{\mu \nu}\left(q_{1}, q_{2}\right)=0, \quad T^{\mu \nu}\left(q_{1}, q_{2}\right) q_{2 \nu}=0
$$

which is indeed the case [68].

Correspondingly, the divergence of the axial-vector current

$$
j_{5}^{\mu}(x)=\bar{\psi}(x) \gamma^{\mu} \gamma^{5} \psi(x)
$$

should be given by

$$
\partial_{\mu} j_{5}^{\mu}(x)=2 m i \bar{\psi}(x) \gamma^{5} \psi(x) .
$$

After applying eq. (A.9) to the definition of $T_{5}^{\mu \nu}\left(q_{1}, q_{2}\right)$ [see eq. (2.13)], we obtain the following Ward-Takahashi identity for the tensor $T_{5}^{\mu \nu}\left(q_{1}, q_{2}\right)$

$$
q_{1 \mu} T_{5}^{\mu \nu}\left(q_{1}, q_{2}\right)=T_{5}^{\nu}\left(q_{1}, q_{2}\right)+\mathfrak{T}_{5}^{\nu}\left(q_{1}, q_{2}\right), \quad T_{5}^{\mu \nu}\left(q_{1}, q_{2}\right) q_{2 \nu}=-\mathfrak{T}_{5}^{\mu}\left(-q_{2},-q_{1}\right),
$$


where we defined

$$
T_{5}^{\nu}\left(q_{1}, q_{2}\right)=2 m \int \frac{d^{4} p d^{4} p^{\prime}}{(2 \pi)^{8}} \operatorname{tr} I\left(p^{\prime}, q_{1}, p\right) \gamma^{5} \frac{\not p+m}{p^{2}-m^{2}+i 0} \Gamma^{\nu}\left(p,-q_{2}, p^{\prime}\right) \frac{\not p^{\prime}+m}{p^{\prime 2}-m^{2}+i 0}
$$

and the so-called anomalous contribution

$$
\begin{aligned}
\mathfrak{T}_{5}^{\nu}\left(q_{1}, q_{2}\right)=\int \frac{d^{4} p d^{4} p^{\prime}}{(2 \pi)^{8}}\left[\operatorname{tr} \Gamma^{\nu}\left(p,-q_{2}, p^{\prime}\right) I\left(p^{\prime}, q_{1}, p\right) \gamma^{5} \frac{\not p+m}{p^{2}-m^{2}+i 0}\right. \\
\left.\quad-\operatorname{tr} I\left(p^{\prime}, q_{1}, p\right) \Gamma^{\nu}\left(p,-q_{2}, p^{\prime}\right) \gamma^{5} \frac{\not p^{\prime}+m}{p^{\prime 2}-m^{2}+i 0}\right] .
\end{aligned}
$$

Furthermore, we introduced the dressed scalar vertex $I\left(p^{\prime}, q, p\right)$ [see eq. (A.4)].

Based on eq. (3.24) one expects that the anomalous contribution to the WardTakahashi identity vanishes. If the relations given in eq. (A.10) are formally applied to eq. (3.27) as in section II.E of [68], it looks like this is really the case. However, a closer analysis reveals that the intermediate expressions are divergent and the required formal manipulations cannot be justified. In fact, as shown in section 4, the anomalous contribution does not vanish and is given by

$$
\mathfrak{T}_{5}^{\nu}\left(q_{1}, q_{2}\right)=-i \pi e^{2} \delta^{(-, \perp)}\left(q_{1}-q_{2}\right) 4 e \int_{-\infty}^{+\infty} d x^{-} e^{i\left(q_{2}^{+}-q_{1}^{+}\right) x^{-}} q_{\mu} F^{* \mu \nu}(k x) .
$$

The phenomenon that quantum fluctuations can spoil the results expected from the classical symmetries of the Lagrangian has first been observed in $[103,104]$ and, as we have mentioned, is known as ABJ anomaly (see also [128-131] for a discussion using the Feynman path integral and e.g. [62, 132, 133] for a textbook discussion).

Unlike the anomalous contribution the calculation of $T_{5}^{\nu}\left(q_{1}, q_{2}\right)$ [see eq. (3.26)] is much less involved. Due to the identity [see eq. (A.4)]

$$
I\left(p^{\prime}, q, p\right) \gamma^{5}=-i e \int d^{4} x\left(\gamma^{5}-\frac{1}{2} G_{3} \mathfrak{F}_{x}^{* \rho \sigma} i \sigma_{\rho \sigma}\right) e^{i S_{\Gamma}\left(p^{\prime}, q, p ; x\right)}
$$

we only have to change the trace in eq. (3.5) to

$$
\begin{aligned}
\frac{1}{4} \operatorname{tr}[\cdots]_{5}^{\nu} & =\frac{1}{4} \operatorname{tr} 2 m\left(\gamma^{5}-\frac{1}{2} G_{3} \mathfrak{F}_{x}^{* \rho \sigma} i \sigma_{\rho \sigma}\right)(\not p+m)\left(\gamma_{\beta} c^{\beta \nu}+i \gamma_{\beta} \gamma^{5} d^{\beta \nu}\right)\left(\not p^{\prime}+m\right) \\
& =2 i m^{2}\left[\left(p-p^{\prime}\right)_{\beta} d^{\beta \nu}+G_{3}\left(p-p^{\prime}\right)^{\alpha} \mathfrak{F}_{x \alpha \beta}^{*} c^{\beta \nu}-G_{3}\left(p+p^{\prime}\right)^{\alpha} \mathfrak{F}_{x \alpha \beta} d^{\beta \nu}\right] .
\end{aligned}
$$

Since the action of the derivatives on $k j$ and $k j^{\prime}$ gives no contribution, we obtain the replacement rules [see eq. (3.10)]

$$
\begin{aligned}
& p^{\mu} \longrightarrow(-i) \partial_{j}^{\mu} \longrightarrow-\frac{1}{s+t}\left(t q_{2}^{\mu}+\frac{1}{2} \lambda^{\mu}\right), \\
& p^{\prime \mu} \longrightarrow(-i) \partial_{j^{\prime}}^{\mu} \longrightarrow \frac{1}{s+t}\left(s q_{2}^{\mu}-\frac{1}{2} \lambda^{\mu}\right),
\end{aligned}
$$

implying

$$
\left(p-p^{\prime}\right)^{\mu} \longrightarrow-q_{2}^{\mu}, \quad\left(p+p^{\prime}\right)^{\mu} \longrightarrow v q_{2}^{\mu}-\frac{1}{\tau} \lambda^{\mu}
$$


After applying them and noting that terms linear in $v$ vanish [see eq. (3.8)], we can replace the trace in eq. (3.30) by

$$
\frac{1}{4} \operatorname{tr}[\cdots]_{5}^{\nu} \longrightarrow 2 i m^{2} G_{3}\left[\left(\mathfrak{F}_{x}^{*} q\right)^{\nu}-\left(\mathfrak{F}_{y}^{*} q\right)^{\nu}\right]
$$

for the calculation of $T_{5}^{\nu}\left(q_{1}, q_{2}\right)$ [see eq. (3.26)].

\subsection{Tensor structure}

Due to the inhomogeneous Ward-Takahashi identity [see eq. (3.25)] the tensor structure of $T_{5}^{\mu \nu}\left(q_{1}, q_{2}\right)$ is more complicated than that of the polarization tensor $T^{\mu \nu}\left(q_{1}, q_{2}\right)$. Using the complete sets $q_{1}^{\mu}, \mathcal{Q}_{1}^{\mu}, \Lambda_{1}^{* \mu}, \Lambda_{2}^{* \mu}$ and $q_{2}^{\nu}, \mathcal{Q}_{2}^{\nu}, \Lambda_{1}^{* \nu}, \Lambda_{2}^{* \nu}$ (see appendix C for more details), we obtain the following expansion

$$
\begin{aligned}
T_{5}^{\mu \nu}= & \mathfrak{T}_{5}^{\mu \nu}+d_{1} \Lambda_{1}^{* \mu} \Lambda_{2}^{* \nu}+d_{2} \Lambda_{2}^{* \mu} \Lambda_{1}^{* \nu}+d_{3} \Lambda_{1}^{* \mu} \Lambda_{1}^{* \nu} \\
& +d_{4} \Lambda_{2}^{* \mu} \Lambda_{2}^{* \nu}+d_{5} \mathcal{Q}_{1}^{\mu} \mathcal{Q}_{2}^{\nu}+d_{6} \mathcal{Q}_{1}^{\mu} \Lambda_{1}^{* \nu} \\
& +d_{7} \mathcal{Q}_{1}^{\mu} \Lambda_{2}^{* \nu}+d_{8} \Lambda_{1}^{* \mu} \mathcal{Q}_{2}^{\nu}+d_{9} \Lambda_{2}^{* \mu} \mathcal{Q}_{2}^{\nu} \\
& +d_{10} q_{1}^{\mu} \Lambda_{1}^{* \nu}+d_{11} q_{1}^{\mu} \Lambda_{2}^{* \nu}+d_{12} q_{1}^{\mu} \mathcal{Q}_{2}^{\nu}
\end{aligned}
$$

where $\mathfrak{T}_{5}^{\mu \nu}\left(q_{1}, q_{2}\right)$ contains the contribution from the anomaly, i.e. [see eq. (3.28)]

$$
\begin{aligned}
\mathfrak{T}_{5}^{\mu \nu}\left(q_{1}, q_{2}\right)= & -i \pi e^{2} \delta^{(-, \perp)}\left(q_{1}-q_{2}\right) 4 e \int_{-\infty}^{+\infty} d x^{-} \\
& \times e^{i\left(q_{2}^{+}-q_{1}^{+}\right) x^{-}} \frac{1}{k q}\left[k^{\mu}\left(q F^{*}\right)^{\nu}(k x)+\left(q F^{*}\right)^{\mu}(k x) k^{\nu}\right] .
\end{aligned}
$$

As expected from Furry's theorem [127], the coefficients $d_{1}-d_{5}$ and $d_{12}$ (which contain an even power of the external field tensors $f_{i}^{\mu \nu}$ ) vanish and only $d_{6}-d_{11}$ are different from zero.

\subsection{Determination of the coefficients}

Having determined the contraction of $T_{5}^{\mu \nu}\left(q_{1}, q_{2}\right)$ with $q_{1}^{\mu}$ and $q_{2}^{\mu}$ explicitly, we can restrict us to the contraction from the set $k, \Lambda_{1}^{*}$ and $\Lambda_{2}^{*}$ (or alternatively $k, \Lambda_{1}$ and $\Lambda_{2}$, see appendix $\mathrm{C}$ ) if we analyze the general trace given in eq. (3.5). This means that, in order to complete the calculation of $T_{5}^{\mu \nu}\left(q_{1}, q_{2}\right)$, we can ignore the action of the derivatives on $k j$ and $k j^{\prime}$ and also terms in the trace which are e.g. proportional to $\mathfrak{F}^{\mu \nu}, \mathfrak{F}^{* \mu \nu}, \mathfrak{F}^{2 \mu \nu},\left(\mathfrak{F} \mathfrak{F}^{*}\right)^{\mu \nu}$, $\left(\mathfrak{F}^{*} \mathfrak{F}\right)^{\mu \nu}, \mathfrak{F}^{2 \mu \rho} v_{\rho}, v_{\rho} \mathfrak{F}^{2 \rho \nu}$, where $v^{\mu}$ is an arbitrary four-vector. In particular, we see that the terms $a^{\alpha \mu} d_{\alpha}{ }^{\nu}$ and $b^{\alpha \mu} c_{\alpha}{ }^{\nu}$ can be ignored and therefore also the action of the derivatives on the term in the exponent which is quadratic in the sources. If the derivatives act on the non-quadratic source-terms in the exponent, we obtain the replacement rules

$$
\begin{aligned}
& p^{\alpha} p^{\prime \beta}+p^{\beta} p^{\prime \alpha} \longrightarrow-\frac{2 \mu}{\tau} q_{2}^{\alpha} q_{2}^{\beta}+\frac{1}{2 \tau^{2}} \lambda^{\alpha} \lambda^{\beta}-\frac{v}{2 \tau}\left(q_{2}^{\alpha} \lambda^{\beta}+\lambda^{\alpha} q_{2}^{\beta}\right), \\
& p^{\alpha} p^{\prime \beta}-p^{\beta} p^{\prime \alpha} \longrightarrow \frac{1}{2 \tau}\left(q_{2}^{\alpha} \lambda^{\beta}-q_{2}^{\beta} \lambda^{\alpha}\right) .
\end{aligned}
$$


Finally, we can replace the trace given in eq. (3.5) by (terms linear in $v$ do not contribute after the integration)

$$
\frac{1}{4} \operatorname{tr}[\cdots]_{5}^{\mu \nu} \longrightarrow i\left\{\frac{e}{k q}\left[q_{2}^{\mu}\left(\mathfrak{F}_{y}^{*} q\right)^{\nu}-\left(\mathfrak{F}_{x}^{*} q\right)^{\mu} q_{2}^{\nu}\right]+\frac{1}{2 \tau} \epsilon^{\mu \nu \rho \sigma} q_{2 \rho} \lambda_{\sigma}\right\}
$$

[as long as only contractions with $k$ and $\Lambda_{i} / \Lambda_{i}^{*}$ are considered, which also means that the anomaly does not contribute, see eq. (3.28)].

\subsection{Final result}

Using the relations given in appendix $\mathrm{C}$ we obtain the following representation for the tensor $T_{5}^{\mu \nu}\left(q_{1}, q_{2}\right)$

$$
\begin{aligned}
T_{5}^{\mu \nu}\left(q_{1}, q_{2}\right)= & \mathfrak{T}_{5}^{\mu \nu}\left(q_{1}, q_{2}\right)-i \pi e^{2} \delta^{(-, \perp)}\left(q_{1}-q_{2}\right) \\
& \times \int_{-1}^{+1} d v \int_{0}^{\infty} \frac{d \tau}{\tau} \int_{-\infty}^{+\infty} d z^{-}\left[a_{6} \mathcal{Q}_{1}^{\mu} \Lambda_{1}^{* \nu}+a_{7} \mathcal{Q}_{1}^{\mu} \Lambda_{2}^{* \nu}+a_{8} \Lambda_{1}^{* \mu} \mathcal{Q}_{2}^{\nu}\right. \\
& \left.\quad+a_{9} \Lambda_{2}^{* \mu} \mathcal{Q}_{2}^{\nu}+a_{10} q_{1}^{\mu} \Lambda_{1}^{* \nu}+a_{11} q_{1}^{\mu} \Lambda_{2}^{* \nu}\right] e^{i \Phi}
\end{aligned}
$$

where the anomalous contribution $\mathfrak{T}_{5}^{\mu \nu}\left(q_{1}, q_{2}\right)$ is given in eq. (3.35) and the coefficients read

$$
\begin{array}{ll}
a_{6}=i m \xi_{1}\left[W_{1}+U_{1} \frac{m^{2}}{q_{1}^{2}} \frac{\tau}{\mu}\right] e^{i \tau \beta}, & \\
a_{7}=i m \xi_{2}\left[W_{2}+U_{2} \frac{m^{2}}{q_{1}^{2}} \frac{\tau}{\mu}\right] e^{i \tau \beta}, & \\
a_{8}=-i m \xi_{1} V_{1} e^{i \tau \beta}, & a_{10}=i m \xi_{1} U_{1} \frac{m^{2}}{q_{1}^{2}} \frac{\tau}{\mu} e^{i \tau \beta}, \\
a_{9}=-i m \xi_{2} V_{2} e^{i \tau \beta}, & a_{11}=i m \xi_{2} U_{2} \frac{m^{2}}{q_{1}^{2}} \frac{\tau}{\mu} e^{i \tau \beta} .
\end{array}
$$

Here, the phases are given by

$$
\begin{aligned}
e^{i \Phi} & =\exp \left\{i\left[\left(q_{2}^{+}-q_{1}^{+}\right) z^{-}+\mu q_{1} q_{2}-\tau m^{2}\right]\right\} \\
e^{i \tau \beta} & =\exp \left[i \tau m^{2} \sum_{i=1,2} \xi_{i}^{2}\left(I_{i}^{2}-J_{i}\right)\right]
\end{aligned}
$$

where [see eq. (3.12)]

$$
I_{i}=\frac{1}{2} \int_{-1}^{+1} d \lambda \psi_{i}(k z-\lambda \mu k q), \quad J_{i}=\frac{1}{2} \int_{-1}^{+1} d \lambda \psi_{i}^{2}(k z-\lambda \mu k q) .
$$

In the preexponent we have introduced the following symbols

$$
U_{i}=\psi_{i}(k x)-\psi_{i}(k y), \quad V_{i}=\psi_{i}(k x)-I_{i}, \quad W_{i}=\psi_{i}(k y)-I_{i},
$$

where $k x=k z-\mu k q, k y=k z+\mu k q$ and $\mu=\frac{1}{4} \tau\left(1-v^{2}\right)$. 
Alternatively, the result in eq. (3.38) can be written as

$$
\begin{aligned}
T_{5}^{\mu \nu}\left(q_{1}, q_{2}\right)= & \mathfrak{T}_{5}^{\mu \nu}\left(q_{1}, q_{2}\right)-i \pi e^{2} \delta^{(-, \perp)}\left(q_{1}-q_{2}\right) \\
& \times \int_{-1}^{+1} d v \int_{0}^{\infty} \frac{d \tau}{\tau} \int_{-\infty}^{+\infty} d z^{-}\left[a_{6}^{\prime} \mathcal{Q}_{1}^{\mu} \Lambda_{1}^{* \nu}+a_{7}^{\prime} \mathcal{Q}_{1}^{\mu} \Lambda_{2}^{* \nu}+a_{8} \Lambda_{1}^{* \mu} \mathcal{Q}_{2}^{\nu}\right. \\
& \left.+a_{9} \Lambda_{2}^{* \mu} \mathcal{Q}_{2}^{\nu}+a_{10}^{\prime} k^{\mu} \Lambda_{1}^{* \nu}+a_{11}^{\prime} k^{\mu} \Lambda_{2}^{* \nu}\right] e^{i \Phi}
\end{aligned}
$$

where

$$
\begin{aligned}
a_{6}^{\prime} & =i m \xi_{1} W_{1} e^{i \tau \beta}, & a_{8}=-i m \xi_{1} V_{1} e^{i \tau \beta}, \\
a_{7}^{\prime} & =i m \xi_{2} W_{2} e^{i \tau \beta}, & a_{9}=-i m \xi_{2} V_{2} e^{i \tau \beta}, \\
a_{10}^{\prime} & =i m \xi_{1} U_{1} \frac{m^{2}}{k q} \frac{\tau}{\mu} e^{i \tau \beta}, & \\
a_{11}^{\prime} & =i m \xi_{2} U_{2} \frac{m^{2}}{k q} \frac{\tau}{\mu} e^{i \tau \beta} . &
\end{aligned}
$$

The last two terms $\left(a_{10}^{\prime}\right.$ and $\left.a_{11}^{\prime}\right)$ are responsible for the inhomogeneous Ward-Takahashi identity [see eq. (3.25) and eq. (3.26)].

\section{Adler-Bell-Jackiw anomaly}

We will show now explicitly that the anomalous contribution $\mathfrak{T}_{5}^{\nu}\left(q_{1}, q_{2}\right)$ [see eq. (3.27)] to the Ward-Takahashi identity for the current-coupling tensor $T_{5}^{\mu \nu}\left(q_{1}, q_{2}\right)$ [see eq. (3.25)] is different from zero.

As pointed out in section 3.1, the formal application of the relations given in eq. (A.10) to eq. (3.27) would prove that $\mathfrak{T}_{5}^{\nu}\left(q_{1}, q_{2}\right)=0$. However, this procedure leads to divergent expressions and a more careful analysis reveals that the obtained result would be incorrect. To determine the anomalous contribution we rewrite eq. (3.27) as

$$
\begin{aligned}
\mathfrak{T}_{5}^{\nu}\left(q_{1}, q_{2}\right)= & \lim _{\epsilon \rightarrow 0} 4(-i e)^{2} \int \frac{d^{4} p d^{4} p^{\prime}}{(2 \pi)^{8}} \int d^{4} x d^{4} y \\
& \times(-i) \int_{0}^{\infty} d s[\underbrace{\frac{1}{4} \operatorname{tr}[\cdots]_{5 A}^{\nu} e^{i \tilde{S}_{T}}}_{t=\epsilon}-\underbrace{\frac{1}{4} \operatorname{tr}[\cdots]_{5 B}^{\nu} e^{i \tilde{S}_{T}}}_{s=\epsilon, t=s}],
\end{aligned}
$$

where the phase $\tilde{S}_{T}$ is defined in eq. (3.14) and the traces are given by

$$
\begin{aligned}
& \frac{1}{4} \operatorname{tr}[\cdots]_{5 A}^{\nu}=i G_{3}\left[\left(p_{\mu} \mathfrak{F}_{x}^{* \mu \nu}-p_{\mu} \mathfrak{F}_{y}^{* \mu \nu}\right)+G_{1} p^{\rho} \mathfrak{F}_{x \rho \mu}^{*} \mathfrak{F}_{y}^{\mu \nu}+G_{3} p^{\rho} \mathfrak{F}_{x \rho \mu} \mathfrak{F}_{y}^{* \mu \nu}\right] \\
& \frac{1}{4} \operatorname{tr}[\cdots]_{5 B}^{\nu}=i G_{3}\left[\left(p_{\mu}^{\prime} \mathfrak{F}_{x}^{* \mu \nu}-p_{\mu}^{\prime} \mathfrak{F}_{y}^{* \mu \nu}\right)+G_{1} p^{\rho \rho} \mathfrak{F}_{x \rho \mu}^{*} \mathfrak{F}_{y}^{\mu \nu}-G_{3} p^{\prime \rho} \mathfrak{F}_{x \rho \mu} \mathfrak{F}_{y}^{* \mu \nu}\right]
\end{aligned}
$$

with $G_{i}=G_{i}\left(k p^{\prime}, k p\right)$.

Although we need to exponentiate here only one scalar propagator [see eq. (3.27)], we artificially add a second term in the exponent (multiplied by a quantity $\epsilon$ which will be later sent to zero), in order to recover exactly the same structure as in eq. (3.14). Also note that the traces in eq. (4.2a) and eq. (4.2b) can be formally obtained from eq. (3.30) by 
setting $p^{\prime \mu}=0$ and $p^{\mu}=0$, respectively, and by dividing by $2 m^{2}$ and $-2 m^{2}$, respectively. To match the first and the second contribution in eq. (4.1), we changed the name of the integration variable $t \rightarrow s$ (after the replacement $s \rightarrow \epsilon$ ) in the second expression.

In order to determine the first and the second contribution to eq. (4.1), respectively, we need to apply the following replacements to eq. (3.13)

$$
\begin{aligned}
& (-i) \int_{0}^{\infty} d t \rightarrow 1, \quad t \rightarrow \epsilon, \quad \operatorname{tr}[\cdots]_{5}^{\mu \nu} \rightarrow \operatorname{tr}[\cdots]_{5 A}^{\nu}, \\
& (-i) \int_{0}^{\infty} d s \rightarrow 1, \quad \begin{array}{l}
s \rightarrow \epsilon, \\
t \rightarrow s,
\end{array} \quad \operatorname{tr}[\cdots]_{5}^{\mu \nu} \rightarrow \operatorname{tr}[\cdots]_{5 B}^{\nu} .
\end{aligned}
$$

In this way, the final result can then be obtained from eq. (3.15).

The replacements given in eq. (4.3) imply that $\tau=s+t$ and $\mu=s t /(s+t)$ are mapped to the same quantity in both expressions, but $v=(s-t) /(s+t)$ changes its sign

$$
\begin{aligned}
& \tau(t \rightarrow \epsilon)=\tau(s \rightarrow \epsilon, t \rightarrow s)=s+\epsilon, \\
& \mu(t \rightarrow \epsilon)=\mu(s \rightarrow \epsilon, t \rightarrow s)=\frac{s \epsilon}{s+\epsilon}, \\
& v(t \rightarrow \epsilon)=-v(s \rightarrow \epsilon, t \rightarrow s)=\frac{s-\epsilon}{s+\epsilon} .
\end{aligned}
$$

We note that due to the relation $k y=k x+2 \mu k q$ [see eq. (57) in [68]], the distance (here in phase) between the two vertices tends to zero as $\epsilon \rightarrow 0$. A similar regularization procedure for the axial-vector vertex is also commonly used in the calculation of the vacuum amplitude (see e.g. chapter 19 of [132] for a textbook discussion).

To use eq. (3.15) we have to apply the replacement rules given in eq. (3.31). Firstly, we note that $\lambda^{\mu}$ does not contribute, as it would only give a non-vanishing contribution after contraction with the first line of each trace in eq. (4.2) which then cancel pairwise. Correspondingly, we can focus on the contribution due to $q_{2}^{\mu}$. As the second and the third line of each trace cancel both pairwise, we focus on the first line. Using the following representation for the delta function

$$
\lim _{\epsilon \rightarrow 0} \int_{0}^{\infty} d x \frac{\epsilon g(x)}{(x+\epsilon)^{2}}=\lim _{\epsilon \rightarrow 0} \int_{0}^{\infty} d y \frac{g(\epsilon y)}{(y+1)^{2}}=g(0)
$$

$[g(x)$ is assumed to be sufficiently regular], we finally obtain the result given in eq. (3.28).

\section{Special field configurations}

In this section the general expression given in eqs. (3.38) and (3.44) is used to derive compact representations for the current-coupling tensor $T_{5}^{\mu \nu}\left(q_{1}, q_{2}\right)$ for three important special field configurations: a constant-crossed field, a relativistically strong, linearly polarized plane-wave background field (quasistatic limit) and a monochromatic, circularly polarized plane-wave background field. When possible, the result is compared with existing representations from the literature. 


\subsection{Constant-crossed field}

From eq. (3.38) we can derive the result for a constant-crossed field, which is characterized by

$$
\psi_{1}(\phi)=\phi, \quad \psi_{2}=0,
$$

(the latter condition corresponds to $\xi_{2}=0$ and we will write $\xi=\xi_{1}$ in the following). For a constant-crossed field the field tensor is given by

$$
F^{\mu \nu}=f_{1}^{\mu \nu}=f^{\mu \nu} .
$$

Since $\psi_{2}=0$, the following functions vanish

$$
I_{2}=J_{2}=U_{2}=V_{2}=W_{2}=0
$$

and due to the simple form of $\psi_{1}$

$$
\begin{aligned}
& I_{1}=k x+\mu k q, \quad J_{1}=(k x+\mu k q)^{2}+\frac{1}{3}(\mu k q)^{2}, \\
& U_{1}=-2 \mu k q, \quad V_{1}=-\mu k q, \quad W_{1}=\mu k q .
\end{aligned}
$$

Finally, we obtain the following explicit expression for the tensor $T_{5}^{\mu \nu}\left(q_{1}, q_{2}\right)$ in a constantcrossed field

$$
\begin{aligned}
T_{5}^{\mu \nu}\left(q_{1}, q_{2}\right)= & \mathfrak{T}_{5}^{\mu \nu}\left(q_{1}, q_{2}\right)-2 i \pi^{2} e^{2} \delta^{4}\left(q_{1}-q_{2}\right) \\
& \times \int_{-1}^{+1} d v \int_{0}^{\infty} \frac{d \tau}{\tau}\left[\tilde{b}_{6}^{\mathrm{c}} \mathcal{Q}^{\mu} \Lambda_{1}^{* \nu}+\tilde{b}_{8}^{\mathrm{c}} \Lambda_{1}^{* \mu} \mathcal{Q}^{\nu}+\tilde{b}_{10}^{\mathrm{c}} q^{\mu} \Lambda_{1}^{* \nu}\right] e^{i \Phi_{\mathrm{c}}}
\end{aligned}
$$

where the coefficients are given by

$$
\begin{aligned}
\tilde{b}_{6}^{\mathrm{c}} & =i \xi m k q\left[\frac{1}{w}-\frac{2 m^{2}}{q^{2}}\right] \tau e^{i \tau \beta_{\mathrm{c}}}, \\
\tilde{b}_{8}^{\mathrm{c}} & =i \xi m k q \frac{1}{w} \tau e^{i \tau \beta_{\mathrm{c}}} \\
\tilde{b}_{10}^{\mathrm{c}} & =i \xi m k q\left(-2 m^{2} / q^{2}\right) \tau e^{i \tau \beta_{\mathrm{c}}},
\end{aligned}
$$

the phases read

$$
\begin{aligned}
i \Phi_{\mathrm{c}} & =-i \tau a, \quad a=m^{2}\left[1-\frac{1}{4}\left(1-v^{2}\right) \frac{q^{2}}{m^{2}}\right], \\
i \tau \beta_{\mathrm{c}} & =-\frac{i}{3} \tau^{3} b, \quad b=m^{6} \chi^{2}\left[\frac{1}{4}\left(1-v^{2}\right)\right]^{2}
\end{aligned}
$$

and the anomaly $\mathfrak{T}_{5}^{\mu \nu}\left(q_{1}, q_{2}\right)$ [see eq. (3.35)] becomes

$$
\mathfrak{T}_{5}^{\mu \nu}\left(q_{1}, q_{2}\right)=i(2 \pi)^{4} \delta^{4}\left(q_{1}-q_{2}\right)\left(-\frac{e^{3}}{8 \pi^{2} m^{2}}\right) 4 \frac{m^{2}}{k q}\left[k^{\mu}\left(q F^{*}\right)^{\nu}+\left(q F^{*}\right)^{\mu} k^{\nu}\right] .
$$

Above, we introduced the quantum-nonlinearity parameter

$$
\chi=-\frac{e \sqrt{q F^{2} q}}{m^{3}}=\xi \frac{\sqrt{(k q)^{2}}}{m^{2}} .
$$


Due to the overall momentum-conserving delta function we define

$$
q^{\mu}=q_{1}^{\mu}=q_{2}^{\mu}, \quad \mathcal{Q}^{\mu}=\mathcal{Q}_{1}^{\mu}=\mathcal{Q}_{2}^{\mu}=\frac{k^{\mu} q^{2}-q^{\mu} k q}{k q} .
$$

Using the relation

$$
\tilde{b}_{6}^{\mathrm{c}} \mathcal{Q}^{\mu} \Lambda_{1}^{* \nu}+\tilde{b}_{10}^{\mathrm{c}} q^{\mu} \Lambda_{1}^{* \nu}=\frac{e}{\xi m k q}\left(\tilde{b}_{6}^{\mathrm{c}}-\tilde{b}_{10}^{\mathrm{c}}\right) q^{\mu}\left(F^{*} q\right)^{\nu}-\frac{e}{\xi m k q} \frac{q^{2}}{k q} \tilde{b}_{6}^{\mathrm{c}} k^{\mu}\left(F^{*} q\right)^{\nu}
$$

we can rewrite eq. (5.5) as

$$
\begin{aligned}
T_{5}^{\mu \nu}\left(q_{1}, q_{2}\right)= & \mathfrak{T}_{5}^{\mu \nu}\left(q_{1}, q_{2}\right)+i(2 \pi)^{4} \delta^{4}\left(q_{1}-q_{2}\right) \\
& \times\left[\tilde{\tau}_{1} \mathcal{Q}^{\mu}\left(F^{*} q\right)^{\nu}+\tilde{\tau}_{2} k^{\mu}\left(F^{*} q\right)^{\nu}+\tilde{\tau}_{1}\left(F^{*} q\right)^{\mu} \mathcal{Q}^{\nu}\right],
\end{aligned}
$$

where

$$
\begin{aligned}
& \tilde{\tau}_{1}=+\frac{e^{3}}{8 \pi^{2} m^{2}} \int_{-1}^{+1} d v \frac{1}{w}\left(\frac{w}{\chi}\right)^{2 / 3} f(\rho), \\
& \tilde{\tau}_{2}=-\frac{e^{3}}{8 \pi^{2} m^{2}} \int_{-1}^{+1} d v 2 \frac{m^{2}}{k q}\left(\frac{w}{\chi}\right)^{2 / 3} f(\rho)
\end{aligned}
$$

$\left[\frac{1}{w}=\frac{1}{4}\left(1-v^{2}\right), \rho=\left(\frac{w}{\chi}\right)^{2 / 3}\left(1-\frac{q^{2}}{m^{2}} \frac{1}{w}\right)\right]$ and the anomaly is given in eq. (5.8). Furthermore, the Ritus functions are defined by $[64,134]$

$$
\begin{aligned}
f(x) & =i \int_{0}^{\infty} d t \exp \left[-i\left(t x+t^{3} / 3\right)\right]=\pi \mathrm{Gi}(x)+i \pi \operatorname{Ai}(x), \\
f_{1}(x) & =\int_{0}^{\infty} \frac{d t}{t} \exp (-i t x)\left[\exp \left(-i t^{3} / 3\right)-1\right]
\end{aligned}
$$

where Ai and Gi are the Airy and Scorer function, respectively [135]. Note that in Ritus' work the normalization of the Airy function is different and also changes [see [64], appendix $\mathrm{C}$ and [136], eq. (B5)].

Since all nonvanishing functions are even in $v$, it is possible to apply the following change of variables

$$
\int_{-1}^{+1} d v=2 \int_{0}^{1} d v=\int_{4}^{\infty} d w \frac{4}{w \sqrt{w(w-4)}}
$$

The final result given in eq. (5.12) coincides with the one given in eq. (4.24) of [99], apart from the anomalous contribution in the vector index [see eq. (3.25)], which automatically drops out by performing the calculations within the wordline formalism as in [99]. If evaluated on the mass shell (i.e. for $q^{2}=0$ ), it also agrees with eq. (15) in [97].

\subsection{Linear polarization}

We consider now a linearly polarized plane-wave field

$$
\psi_{1}(\phi)=\psi(\phi), \quad \psi_{2}=0
$$


$\left(\xi=\xi_{1}, f^{\mu \nu}=f_{1}^{\mu \nu}\right)$ in the quasistatic limit defined by $\xi \rightarrow \infty$ while [see eq. (5.9)]

$$
\chi=-\frac{e \sqrt{q f^{2} q}}{m^{3}}=\xi \frac{\sqrt{(k q)^{2}}}{m^{2}}
$$

is kept constant. In the optical regime (photon energy $\omega_{0} \sim 1 \mathrm{eV}$ ) the condition $\chi \gtrsim 1$ usually requires $\xi \gg 1$, which means that the quasistatic limit is in general sufficient to analyze strong-field experiments with optical lasers (it neglects the recollision contribution considered in [137], though).

For a linearly polarized background field we obtain

$$
I_{2}=J_{2}=U_{2}=V_{2}=W_{2}=0
$$

and, using the relation $|k q|=m^{2} \chi / \xi$, it is sufficient to consider the leading-order contribution to the following quantities

$$
\begin{aligned}
I_{1}^{2}-J_{1} & =-(1 / 3)(\mu k q)^{2}\left[\psi^{\prime}(k z)\right]^{2}+\mathcal{O}(\mu k q)^{3}, \\
U_{1} & =-2 \mu k q \psi^{\prime}(k z)+\mathcal{O}(\mu k q)^{2}, \\
V_{1} & =-\mu k q \psi^{\prime}(k z)+\mathcal{O}(\mu k q)^{2}, \\
W_{1} & =+\mu k q \psi^{\prime}(k z)+\mathcal{O}(\mu k q)^{2} .
\end{aligned}
$$

If we insert these relations into eq. (3.44), the remaining calculation is very similar to the one for a constant-crossed field (see section 5.1), the essential change is the replacement $\chi \rightarrow \chi(k z)=\chi\left|\psi^{\prime}(k z)\right|$. The final result is given by

$$
\begin{aligned}
T_{5}^{\mu \nu}\left(q_{1}, q_{2}\right)= & \mathfrak{T}_{5}^{\mu \nu}\left(q_{1}, q_{2}\right)+i(2 \pi)^{4} \delta^{(-, \perp)}\left(q_{1}-q_{2}\right) \\
& \times \frac{1}{2 \pi} \int_{-\infty}^{+\infty} d z^{-} e^{i\left(q_{2}^{+}-q_{1}^{+}\right) z^{-}} \psi^{\prime}(k z)\left[\tau_{1}^{\prime} \mathcal{Q}_{1}^{\mu}\left(f^{*} q\right)^{\nu}+\tau_{1}^{\prime}\left(f^{*} q\right)^{\mu} \mathcal{Q}_{2}^{\nu}+\tau_{2}^{\prime} k^{\mu}\left(f^{*} q\right)^{\nu}\right]
\end{aligned}
$$

where

$$
\begin{aligned}
& \tau_{1}^{\prime}=+\frac{e^{3}}{8 \pi^{2} m^{2}} \int_{-1}^{+1} d v \frac{1}{w}\left[\frac{w}{|\chi(k z)|}\right]^{2 / 3} f(\rho), \\
& \tau_{2}^{\prime}=-\frac{e^{3}}{8 \pi^{2} m^{2}} \int_{-1}^{+1} d v \frac{2 m^{2}}{k q}\left[\frac{w}{|\chi(k z)|}\right]^{2 / 3} f(\rho)
\end{aligned}
$$

and $\frac{1}{w}=\frac{1}{4}\left(1-v^{2}\right), \rho=\left[\frac{w}{|\chi(k z)|}\right]^{2 / 3}\left(1-\frac{q_{1} q_{2}}{m^{2}} \frac{1}{w}\right)$. Furthermore, the anomaly reads $[$ see eq. (3.35)]

$$
\begin{aligned}
\mathfrak{T}_{5}^{\mu \nu}\left(q_{1}, q_{2}\right)= & i(2 \pi)^{4} \delta^{(-, \perp)}\left(q_{1}-q_{2}\right) \frac{1}{2 \pi} \int_{-\infty}^{+\infty} d z^{-} e^{i\left(q_{2}^{+}-q_{1}^{+}\right) z^{-}} \psi^{\prime}(k z) \\
& \times\left(-\frac{e^{3}}{8 \pi^{2} m^{2}}\right) 4 \frac{m^{2}}{k q}\left[k^{\mu}\left(q f^{*}\right)^{\nu}+\left(q f^{*}\right)^{\mu} k^{\nu}\right] .
\end{aligned}
$$

Note that for $\psi^{\prime}(\phi)=1$ the result given in eq. (5.20) coincides (as required) with the one for a constant-crossed field [see eq. (5.12)]. 


\subsection{Circular polarization}

Also for a circularly polarized, monochromatic background field

$$
\psi_{1}(\phi)=\operatorname{Re} e^{i \phi}, \quad \psi_{2}(\phi)=\operatorname{Im} e^{i \phi}, \quad \xi_{1}=\xi_{2}=\xi
$$

the result given in eq. (3.44) simplifies considerably and we obtain

$$
\begin{aligned}
T_{5}^{\mu \nu}\left(q_{1}, q_{2}\right)= & \mathfrak{T}_{5}^{\mu \nu}\left(q_{1}, q_{2}\right)-i \pi e^{2} \delta^{(-, \perp)}\left(q_{1}-q_{2}\right) \\
& \times \int_{-1}^{+1} d v \int_{0}^{\infty} \frac{d \tau}{\tau} \int_{-\infty}^{+\infty} d z^{-}\left[a_{1}^{+} \mathcal{Q}_{1}^{\mu} \widetilde{\Lambda}_{+}^{\nu}+a_{1}^{-} \mathcal{Q}_{1}^{\mu} \widetilde{\Lambda}_{-}^{\nu}+a_{2}^{+} \widetilde{\Lambda}_{+}^{\mu} \mathcal{Q}_{2}^{\nu}\right. \\
& \left.+a_{2}^{-} \widetilde{\Lambda}_{-}^{\mu} \mathcal{Q}_{2}^{\nu}+a_{3}^{+} k^{\mu} \widetilde{\Lambda}_{+}^{\nu}+a_{3}^{-} k^{\mu} \widetilde{\Lambda}_{-}^{\nu}\right] e^{i \Phi}
\end{aligned}
$$

where the anomaly is given in eq. (3.35) and

$$
\begin{aligned}
a_{1}^{+}= & \frac{1}{2}\left(a_{6}^{\prime}-i a_{7}^{\prime}\right)=\frac{1}{2} i m \xi\left(W_{1}-i W_{2}\right) e^{i \tau \beta}, \\
a_{1}^{-}= & \frac{1}{2}\left(a_{6}^{\prime}+i a_{7}^{\prime}\right)=\frac{1}{2} i m \xi\left(W_{1}+i W_{2}\right) e^{i \tau \beta}, \\
a_{2}^{+}= & \frac{1}{2}\left(a_{8}-i a_{9}\right)=-\frac{1}{2} i m \xi\left(V_{1}-i V_{2}\right) e^{i \tau \beta}, \\
a_{2}^{-}= & \frac{1}{2}\left(a_{8}+i a_{9}\right)=-\frac{1}{2} i m \xi\left(V_{1}+i V_{2}\right) e^{i \tau \beta}, \\
a_{3}^{+}= & \frac{1}{2}\left(a_{10}^{\prime}-i a_{11}^{\prime}\right)=\frac{1}{2} i m \xi \frac{\tau m^{2}}{\mu k q}\left(U_{1}-i U_{2}\right) e^{i \tau \beta}, \\
a_{3}^{-}= & \frac{1}{2}\left(a_{10}^{\prime}+i a_{11}^{\prime}\right)=\frac{1}{2} i m \xi \frac{\tau m^{2}}{\mu k q}\left(U_{1}+i U_{2}\right) e^{i \tau \beta}, \\
& i \tau \beta=i \tau m^{2} \xi^{2}\left[\operatorname{sinc}^{2}(\mu k q)-1\right], \\
& i \Phi=i\left[\left(q_{2}^{+}-q_{1}^{+}\right) z^{-}+\mu q_{1} q_{2}-\tau m^{2}\right]
\end{aligned}
$$

and

$$
\widetilde{\Lambda}_{ \pm}^{\mu}=\Lambda_{1}^{* \mu} \pm i \Lambda_{2}^{* \mu}
$$

(the star is part of the symbol, both $\Lambda_{1}^{* \mu}$ and $\Lambda_{2}^{* \mu}$ are real four-vectors). Furthermore,

$$
\begin{aligned}
W_{1}+i W_{2} & =-A, & W_{1}-i W_{2} & =-A^{*}, \\
V_{1}+i V_{2} & =-B, & V_{1}-i V_{2} & =-B^{*}, \\
U_{1}+i U_{2} & =-C, & U_{1}-i U_{2} & =-C^{*}
\end{aligned}
$$

where

$$
\begin{aligned}
& A=e^{i k z}[\operatorname{sinc}(\mu k q)-\cos (\mu k q)-i \sin (\mu k q)], \\
& B=e^{i k z}[\operatorname{sinc}(\mu k q)-\cos (\mu k q)+i \sin (\mu k q)], \\
& C=e^{i k z} 2 i \sin (\mu k q)
\end{aligned}
$$


and therefore

$$
\begin{aligned}
-W_{1} & =I_{1}-\psi_{1}(k z+\mu k q)=\operatorname{Re} A, \\
-W_{2} & =I_{2}-\psi_{2}(k z+\mu k q)=\operatorname{Im} A, \\
-V_{1} & =I_{1}-\psi_{1}(k z-\mu k q)=\operatorname{Re} B, \\
-V_{2} & =I_{2}-\psi_{2}(k z-\mu k q)=\operatorname{Im} B, \\
-U_{1} & =\psi_{1}(k z+\mu k q)-\psi_{1}(k z-\mu k q)=\operatorname{Re} C, \\
-U_{2} & =\psi_{2}(k z+\mu k q)-\psi_{2}(k z-\mu k q)=\operatorname{Im} C .
\end{aligned}
$$

We can now take the integral in $d z^{-}$and obtain

$$
\begin{aligned}
T_{5}^{\mu \nu}\left(q_{1}, q_{2}\right)= & \mathfrak{T}_{5}^{\mu \nu}\left(q_{1}, q_{2}\right)-i(2 \pi)^{4} \frac{e^{2}}{8 \pi^{2}} \\
& \times \int_{-1}^{+1} d v \int_{0}^{\infty} \frac{d \tau}{\tau}\left[T_{5+}^{\mu \nu} \delta\left(q_{1}-q_{2}+k\right)+T_{5-}^{\mu \nu} \delta\left(q_{1}-q_{2}-k\right)\right] e^{i \Phi_{\mathrm{cp}}},
\end{aligned}
$$

where

$$
\begin{gathered}
i \Phi_{\mathrm{cp}}=-i \tau m^{2}\left\{1+\xi^{2}\left[1-\operatorname{sinc}^{2}(\mu k q)\right]\right\}+i \mu q_{1} q_{2}, \\
T_{5 \pm}^{\mu \nu}=\left(\lambda_{1}^{ \pm} \mathcal{Q}_{1}^{\mu}+\lambda_{3}^{ \pm} k^{\mu}\right) \widetilde{\Lambda}_{ \pm}^{\nu}+\lambda_{2}^{ \pm} \widetilde{\Lambda}_{ \pm}^{\mu} \mathcal{Q}_{2}^{\nu}
\end{gathered}
$$

and

$$
\begin{aligned}
& \lambda_{1}^{ \pm}=-\frac{1}{2} i m \xi[\operatorname{sinc}(\mu k q)-\cos (\mu k q) \pm i \sin (\mu k q)], \\
& \lambda_{2}^{ \pm}=+\frac{1}{2} i m \xi[\operatorname{sinc}(\mu k q)-\cos (\mu k q) \mp i \sin (\mu k q)], \\
& \lambda_{3}^{ \pm}=\mp m \xi \tau m^{2} \operatorname{sinc}(\mu k q) .
\end{aligned}
$$

Correspondingly, the result is in agreement with the one obtained in [55-57].

\section{Conclusion}

In the present paper the axial-vector-vector current-coupling tensor $T_{5}^{\mu \nu}\left(q_{1}, q_{2}\right)$ [see figure 2 and eq. (2.13)] has been considered for the first time in the presence of a general plane-wave background field (arbitrary polarization and pulse shape). The (pseudo-)tensor $T_{5}^{\mu \nu}\left(q_{1}, q_{2}\right)$ appears in the calculation of various electroweak processes inside strong laser fields like photon emission by neutrinos [see figure 1 and eq. (2.11)]. We derived a triple-integral representation for $T_{5}^{\mu \nu}\left(q_{1}, q_{2}\right)$ [see eqs. (3.38) and (3.44)], which can even be converted into a double-integral representation (see [54] for details). In particular, the anomalous contribution to the Ward-Takahashi identity [see eq. (3.25)] due to the Adler-Bell-Jackiw (ABJ) anomaly associated with the axial-vector current has been calculated explicitly [see figure 3 and eq. (3.28)]. Finally, we specialized the obtained general expression to three important types of background plane waves and confirmed agreement with the corresponding results available in the literature: a constant-crossed field [see eq. (5.12)], a relativistically strong, linearly polarized plane-wave background field [see eq. (5.20)] and a monochromatic, circularly polarized plane-wave background field [see eq. (5.31)].

\section{Acknowledgments}

S.M. is grateful to the Studienstiftung des deutschen Volkes for financial support. 


\section{A Dressed vertex}

A strong plane-wave background field can be taken into account exactly by using dressed states for the charged particles. In momentum space this implies that the free vertex must be replaced by the so-called dressed vertex (see e.g. [65, 68] for more details). Using the Ritus $E_{p}$ matrices [64, 65]

$$
E_{p, x}=\left[\mathbf{1}+\frac{e \not k A(k x)}{2 k p}\right] e^{i S_{p}(x)}, \quad \bar{E}_{p, x}=\left[\mathbf{1}+\frac{e \not A(k x) \not k}{2 k p}\right] e^{-i S_{p}(x)}
$$

which contain the Volkov action

$$
S_{p}(x)=-p x-\int_{-\infty}^{k x}\left[\frac{e p A\left(\phi^{\prime}\right)}{k p}-\frac{e^{2} A^{2}\left(\phi^{\prime}\right)}{2 k p}\right] d \phi^{\prime},
$$

we define the dressed vector $\left(\Gamma^{\rho}\right)$ and scalar $(I)$ vertices by

$$
\begin{aligned}
\Gamma^{\rho}\left(p^{\prime}, q, p\right) & =-i e \int d^{4} x e^{-i q x} \bar{E}_{p^{\prime}, x} \gamma^{\rho} E_{p, x}, \\
I\left(p^{\prime}, q, p\right) & =-i e \int d^{4} x e^{-i q x} \bar{E}_{p^{\prime}, x} E_{p, x} .
\end{aligned}
$$

They can be decomposed in the following way [68]

$$
\begin{aligned}
\Gamma^{\rho}\left(p^{\prime}, q, p\right) & =-i e \int d^{4} x\left[\gamma_{\mu} G^{\mu \rho}\left(k p^{\prime}, k p ; k x\right)+i \gamma_{\mu} \gamma^{5} G_{5}^{\mu \rho}\left(k p^{\prime}, k p ; k x\right)\right] e^{i S_{\Gamma}}, \\
I\left(p^{\prime}, q, p\right) & =-i e \int d^{4} x\left[\mathbf{1}+\frac{G_{3}}{2} \sigma^{\alpha \beta} \mathfrak{F}_{\alpha \beta}(k x)\right] e^{i S_{\Gamma}},
\end{aligned}
$$

where we introduced the phase

$$
\begin{aligned}
S_{\Gamma} & =S_{\Gamma}\left(p^{\prime}, q, p ; x\right)=-S_{p^{\prime}}(x)-q x+S_{p}(x) \\
& =\left(p^{\prime}-q-p\right) x+\int_{-\infty}^{k x} d \phi^{\prime}\left[\frac{e p_{\mu} p_{\nu}^{\prime} \mathfrak{F}^{\mu \nu}\left(\phi^{\prime}\right)}{(k p)\left(k p^{\prime}\right)}+\frac{e^{2}\left(k p-k p^{\prime}\right)}{2(k p)^{2}\left(k p^{\prime}\right)^{2}} p_{\mu} p_{\nu}^{\prime} \mathfrak{F}^{2 \mu \nu}\left(\phi^{\prime}\right)\right]
\end{aligned}
$$

and the following tensors

$$
\begin{gathered}
G^{\mu \rho}\left(k p^{\prime}, k p ; k x\right)=g^{\mu \rho}+G_{1} \mathfrak{F}_{x}^{\mu \rho}+G_{2} \mathfrak{F}_{x}^{2 \mu \rho}, \\
G_{5}^{\mu \rho}\left(k p^{\prime}, k p ; k x\right)=G_{3} \mathfrak{F}_{x}^{* \mu \rho} \\
G_{1}=G_{1}\left(k p^{\prime}, k p\right)=-e \frac{k p+k p^{\prime}}{2 k p k p^{\prime}} \\
G_{2}=G_{2}\left(k p^{\prime}, k p\right)=\frac{e^{2}}{2 k p k p^{\prime}}, \\
G_{3}=G_{3}\left(k p^{\prime}, k p\right)=-e \frac{k p-k p^{\prime}}{2 k p k p^{\prime}}
\end{gathered}
$$

Finally, we note that the dressed vector and scalar vertices are related by [65]

$$
q_{\rho} \Gamma^{\rho}\left(p^{\prime}, q, p\right)=\left(\not p^{\prime}-m\right) I\left(p^{\prime}, q, p\right)-I\left(p^{\prime}, q, p\right)(\not p-m)
$$


and we obtain

$$
\begin{aligned}
& \int \frac{d^{4} p^{\prime \prime}}{(2 \pi)^{4}} I\left(p, q^{\prime}, p^{\prime \prime}\right) \Gamma^{\mu}\left(p^{\prime \prime}, q, p^{\prime}\right)=-i e \Gamma^{\mu}\left(p, q+q^{\prime}, p^{\prime}\right), \\
& \int \frac{d^{4} p^{\prime \prime}}{(2 \pi)^{4}} \Gamma^{\mu}\left(p, q, p^{\prime \prime}\right) I\left(p^{\prime \prime}, q^{\prime}, p^{\prime}\right)=-i e \Gamma^{\mu}\left(p, q+q^{\prime}, p^{\prime}\right) .
\end{aligned}
$$

\section{B Gamma algebra}

Any $4 \times 4$ matrix $\Gamma$ in spinor space can be decomposed into five fundamental constituents $[68,138]$

$$
\Gamma=c_{\mathbf{1}} \mathbf{1}+c_{5} \gamma^{5}+c_{\mu} \gamma^{\mu}+c_{5 \mu} i \gamma^{\mu} \gamma^{5}+c_{\mu \nu} i \sigma^{\mu \nu}
$$

where

$$
\begin{array}{rlrl}
c_{1} & =\frac{1}{4} \operatorname{tr} 1 \Gamma, & c_{5} & =\frac{1}{4} \operatorname{tr} \gamma^{5} \Gamma, \quad c_{\mu}=\frac{1}{4} \operatorname{tr} \gamma_{\mu} \Gamma, \\
c_{5 \mu} & =\frac{1}{4} \operatorname{tr} i \gamma_{\mu} \gamma^{5} \Gamma, \quad c_{\mu \nu}=\frac{1}{8} \operatorname{tr} i \sigma_{\mu \nu} \Gamma .
\end{array}
$$

Instead of the vector and the axial-vector current one can also use the left- and the righthanded current

$$
c_{\mu} \gamma^{\mu}+c_{5 \mu} i \gamma^{\mu} \gamma^{5}=l_{\mu} \gamma^{\mu} P_{L}+r_{\mu} \gamma^{\mu} P_{R}
$$

where the chirality projectors for the left and the right-handed component are given by

$$
P_{L}=\frac{1}{2}\left(\mathbf{1}+\gamma^{5}\right), \quad P_{R}=\frac{1}{2}\left(\mathbf{1}-\gamma^{5}\right)
$$

(note that we define $\gamma^{5}=-i \gamma^{0} \gamma^{1} \gamma^{2} \gamma^{3}$ as in [67]). The coefficients $c_{\mu}, c_{5 \mu}$ and $l_{\mu}, r_{\mu}$ are related via

$$
l_{\mu}=c_{\mu}+i c_{5 \mu}, \quad r_{\mu}=c_{\mu}-i c_{5 \mu}
$$

and

$$
c_{\mu}=\frac{1}{2}\left(l_{\mu}+r_{\mu}\right), \quad c_{5 \mu}=\frac{i}{2}\left(r_{\mu}-l_{\mu}\right) .
$$

Therefore, the coefficients for the left- and the right-handed current can be determined from the following traces

$$
l_{\mu}=\frac{1}{2} \operatorname{tr} P_{L} \gamma_{\mu} \Gamma, \quad r_{\mu}=\frac{1}{2} \operatorname{tr} P_{R} \gamma_{\mu} \Gamma .
$$

Finally, we note the following contraction identities

$$
\begin{array}{rlrl}
\gamma^{\rho} \mathbf{1} \gamma_{\rho} & =4, & \gamma^{\rho} \gamma^{5} \gamma_{\rho} & =-4 \gamma^{5}, \\
\gamma^{\rho} \gamma^{\mu} \gamma_{\rho} & =-2 \gamma^{\mu}, & \gamma^{\rho}\left(i \gamma^{\mu} \gamma^{5}\right) \gamma_{\rho}=2\left(i \gamma^{\mu} \gamma^{5}\right), \\
\gamma^{\rho}\left(i \sigma^{\mu \nu}\right) \gamma_{\rho} & =0 . & &
\end{array}
$$




\section{Summary of important relations}

To obtain a simple structure we expanded the polarization tensor $T^{\mu \nu}\left(q_{1}, q_{2}\right)$ using the two complete sets $q_{1}^{\mu}, \mathcal{Q}_{1}^{\mu}, \Lambda_{1}^{\mu}, \Lambda_{2}^{\mu}$ and $q_{2}^{\nu}, \mathcal{Q}_{2}^{\nu}, \Lambda_{1}^{\nu}, \Lambda_{2}^{\nu}$ [93], where

$$
\begin{aligned}
\Lambda_{1}^{\mu} & =\frac{f_{1}^{\mu \nu} q_{\nu}}{k q \sqrt{-a_{1}^{2}}}, & \Lambda_{2}^{\mu} & =\frac{f_{2}^{\mu \nu} q_{\nu}}{k q \sqrt{-a_{2}^{2}}}, \\
\mathcal{Q}_{1}^{\mu} & =\frac{k^{\mu} q_{1}^{2}-q_{1}^{\mu} k q}{k q}, & \mathcal{Q}_{2}^{\mu} & =\frac{k^{\mu} q_{2}^{2}-q_{2}^{\mu} k q}{k q} .
\end{aligned}
$$

They have the following properties

$$
\begin{aligned}
\Lambda_{i} \Lambda_{j} & =-\delta_{i j}, & k \Lambda_{i} & =q_{i} \Lambda_{j}=\mathcal{Q}_{i} \Lambda_{j}=0 \\
\mathcal{Q}_{1}^{2} & =-q_{1}^{2}, \quad & \mathcal{Q}_{2}^{2} & =-q_{2}^{2},
\end{aligned}
$$

Since $T_{5}^{\mu \nu}\left(q_{1}, q_{2}\right)$ includes $\mathfrak{F}^{* \mu \nu}$ [while $T^{\mu \nu}\left(q_{1}, q_{2}\right)$ contains $\mathfrak{F}^{\mu \nu}$ ] it is more natural to expand $T_{5}^{\mu \nu}\left(q_{1}, q_{2}\right)$ using the two complete sets $q_{1}^{\mu}, \mathcal{Q}_{1}^{\mu}, \Lambda_{1}^{* \mu}, \Lambda_{2}^{* \mu}$ and $q_{2}^{\nu}, \mathcal{Q}_{2}^{\nu}, \Lambda_{1}^{* \nu}, \Lambda_{2}^{* \nu}$ where

$$
\Lambda_{1}^{* \mu}=\frac{f_{1}^{* \mu \nu} q_{\nu}}{k q \sqrt{-a_{1}^{2}}}, \quad \Lambda_{2}^{* \mu}=\frac{f_{2}^{* \mu \nu} q_{\nu}}{k q \sqrt{-a_{2}^{2}}} .
$$

The (pseudo) four-vectors $\Lambda_{i}^{* \mu}$ have similar properties as the four-vectors $\Lambda_{i}^{\mu}$ [compare with eq. (C.2)]

$$
\Lambda_{i}^{*} \Lambda_{j}^{*}=-\delta_{i j}, \quad k \Lambda_{i}^{*}=q_{i} \Lambda_{j}^{*}=\mathcal{Q}_{i} \Lambda_{j}^{*}=0 .
$$

Thus, $\Lambda_{i}^{\mu}$ and $\Lambda_{i}^{* \mu}$ span the same subspace and we obtain the following scalar products

$$
\Lambda_{i} \Lambda_{i}^{*}=0, \quad \Lambda_{1}^{*} \Lambda_{2}=\frac{q \Lambda_{5}}{k q}, \quad \Lambda_{1} \Lambda_{2}^{*}=-\frac{q \Lambda_{5}}{k q},
$$

where we defined the pseudo four-vector

$$
\Lambda_{5}^{\mu}=\frac{\epsilon^{\mu \nu \rho \sigma} k_{\nu} a_{1 \rho} a_{2 \sigma}}{\sqrt{-a_{1}^{2}} \sqrt{-a_{2}^{2}}}=\frac{q \Lambda_{5}}{k q} k^{\mu},
$$

which obeys

$$
\Lambda_{5}^{2}=\Lambda_{5} k=\Lambda_{5} \Lambda_{1}=\Lambda_{5} \Lambda_{2}=0, \quad\left(q \Lambda_{5}\right)^{2}=(k q)^{2}
$$

[beside being a pseudo four-vector $\Lambda_{5}^{\mu}$ is proportional to $k^{\mu}$ ]. Thus, we obtain the identities

$$
\Lambda_{1}^{\mu}=\frac{k q}{q \Lambda_{5}} \Lambda_{2}^{* \mu}, \quad \Lambda_{2}^{\mu}=-\frac{k q}{q \Lambda_{5}} \Lambda_{1}^{* \mu} .
$$

We note the following relations

$$
\begin{gathered}
f_{i \rho}^{\mu} f_{j}^{\rho \nu}=-\delta_{i j} a_{i}^{2} k^{\mu} k^{\nu}, \quad f_{i}^{* \mu} f_{j}^{* \rho \nu}=-\delta_{i j} a_{i}^{2} k^{\mu} k^{\nu}, \quad f_{i}^{* \mu \rho} f_{i \rho \nu}=f_{i}^{\mu \rho} f_{i \rho \nu}^{*}=0, \\
f_{1}^{\mu \rho} f_{2 \rho}^{* \nu}=\sqrt{-a_{1}^{2}} \sqrt{-a_{2}^{2}} k^{\mu} \Lambda_{5}^{\nu}, \quad f_{1}^{* \mu \rho} f_{2 \rho}{ }^{\nu}=-\sqrt{-a_{1}^{2}} \sqrt{-a_{2}^{2}} \Lambda_{5}^{\mu} k^{\nu}, \\
f_{2}^{\mu \rho} f_{1 \rho}^{* \nu}=-\sqrt{-a_{1}^{2}} \sqrt{-a_{2}^{2}} k^{\mu} \Lambda_{5}^{\nu}, \quad f_{2}^{* \mu \rho} f_{1 \rho}^{\nu}=\sqrt{-a_{1}^{2}} \sqrt{-a_{2}^{2}} \Lambda_{5}^{\mu} k^{\nu}
\end{gathered}
$$


which imply

$$
\begin{aligned}
\mathfrak{F}_{x}^{\mu \nu} \Lambda_{i \nu} & =-\frac{m}{e} k^{\mu} \xi_{i} \psi_{i}(k x), \\
\mathfrak{F}_{x}^{* \mu \nu} \Lambda_{1 \nu} & =-\frac{m}{e} \Lambda_{5}^{\mu} \xi_{2} \psi_{2}(k x), \\
\mathfrak{F}_{x}^{* \mu \nu} \Lambda_{2 \nu} & =\frac{m}{e} \Lambda_{5}^{\mu} \xi_{1} \psi_{1}(k x)
\end{aligned}
$$

and

$$
\begin{aligned}
\mathfrak{F}_{x}^{* \mu \nu} \Lambda_{i \nu}^{*} & =-\frac{m}{e} k^{\mu} \xi_{i} \psi_{i}(k x), \\
\mathfrak{F}_{x}^{\mu \nu} \Lambda_{1 \nu}^{*} & =\frac{m}{e} \frac{q \Lambda_{5}}{k q} k^{\mu} \xi_{2} \psi_{2}(k x), \\
\mathfrak{F}_{x}^{\mu \nu} \Lambda_{2 \nu}^{*} & =-\frac{m}{e} \frac{q \Lambda_{5}}{k q} k^{\mu} \xi_{1} \psi_{1}(k x) .
\end{aligned}
$$

Correspondingly, we obtain the canonical choices $\mathfrak{F} \leftrightarrow \Lambda_{i}$ and $\mathfrak{F}^{*} \leftrightarrow \Lambda_{i}^{*}$ (especially for linearly polarized background fields the appearance of $\Lambda_{5}^{\mu}$ is unnatural, since its definition involves both $a_{1}^{\mu}$ and $a_{2}^{\mu}$ ).

We also note that

$$
\epsilon^{\mu \nu \rho \sigma} k_{\rho} q_{\sigma}=-q \Lambda_{5}\left(\Lambda_{1}^{\mu} \Lambda_{2}^{\nu}-\Lambda_{2}^{\mu} \Lambda_{1}^{\nu}\right)
$$

and

$$
\begin{aligned}
\mathfrak{F}_{x}^{\mu \rho} \mathfrak{F}_{y \rho \nu} & =\frac{m^{2}}{e^{2}} k^{\mu} k_{\nu} \sum_{i=1,2} \xi_{i}^{2} \psi_{i}(k x) \psi_{i}(k y), \\
\mathfrak{F}_{x}^{* \mu \rho} \mathfrak{F}_{y \rho \nu} & =-\frac{m^{2}}{e^{2}} \xi_{1} \xi_{2} \Lambda_{5}^{\mu} k_{\nu}\left[\psi_{1}(k x) \psi_{2}(k y)-\psi_{1}(k y) \psi_{2}(k x)\right], \\
\mathfrak{F}_{x}^{\mu \rho} \mathfrak{F}_{y \rho \nu}^{*} & =\frac{m^{2}}{e^{2}} \xi_{1} \xi_{2} k^{\mu} \Lambda_{5 \nu}\left[\psi_{1}(k x) \psi_{2}(k y)-\psi_{1}(k y) \psi_{2}(k x)\right] .
\end{aligned}
$$

Using these relations we can show that for $j=j^{\prime}=0$

$$
\begin{aligned}
\lambda^{\mu} & =-2 m \tau \sum_{i=1,2} \Lambda_{i}^{\mu} \xi_{i} I_{i}, \\
\mathfrak{F}_{x}^{\mu \nu} \lambda_{\nu} & =2 \frac{m^{2}}{e} \tau k^{\mu} \sum_{i=1,2} \xi_{i}^{2} \psi_{i}(k x) I_{i}, \\
\mathfrak{F}_{x}^{* \mu \nu} \lambda_{\nu} & =-2 \frac{m^{2}}{e} \tau \Lambda_{5}^{\mu} \xi_{1} \xi_{2}\left[\psi_{1}(k x) I_{2}-\psi_{2}(k x) I_{1}\right], \\
\Lambda_{i} \lambda & =2 m \tau \xi_{i} I_{i} \\
e q \mathfrak{F}_{x} \lambda & =2 k q \tau m^{2} \sum_{i=1,2} \xi_{i}^{2} \psi_{i}(k x) I_{i}, \\
\Lambda_{i \mu}^{*} k_{\nu} \epsilon^{\mu \nu \rho \sigma} q_{\rho} \lambda_{\sigma} & =2 m \tau k q \xi_{i} I_{i}, \\
\Lambda_{1 \mu}^{*} \Lambda_{2 \nu}^{*} \epsilon^{\mu \nu \rho \sigma} q_{\rho} \lambda_{\sigma} & =0, \\
\Lambda_{1 \mu} k_{\nu} \epsilon^{\mu \nu \rho \sigma} q_{\rho} \lambda_{\sigma} & =2 m \tau \xi_{2} I_{2} q \Lambda_{5}, \\
\Lambda_{2 \mu} k_{\nu} \epsilon^{\mu \nu \rho \sigma} q_{\rho} \lambda_{\sigma} & =-2 m \tau \xi_{1} I_{1} q \Lambda_{5}, \\
\Lambda_{1 \mu} \Lambda_{2 \nu} \epsilon^{\mu \nu \rho \sigma} q_{\rho} \lambda_{\sigma} & =0
\end{aligned}
$$

and

$$
e \Lambda_{i \mu} \mathfrak{F}_{x}^{\mu \nu} q_{\nu}=m k q \xi_{i} \psi_{i}(k x)
$$


Open Access. This article is distributed under the terms of the Creative Commons Attribution License (CC-BY 4.0), which permits any use, distribution and reproduction in any medium, provided the original author(s) and source are credited.

\section{References}

[1] Particle Data Group collaboration, K.A. Olive et al., Review of Particle Physics, Chin. Phys. C 38 (2014) 090001.

[2] S. Bilenky, Introduction to the Physics of Massive and Mixed Neutrinos, Springer, Berlin Germany (2010).

[3] F.T. Avignone III, S.R. Elliott and J. Engel, Double Beta Decay, Majorana Neutrinos and Neutrino Mass, Rev. Mod. Phys. 80 (2008) 481 [arXiv:0708.1033] [InSPIRE].

[4] C. Giunti and C.W. Kim, Fundamentals of Neutrino Physics and Astrophysics, Oxford University Press, U.S.A., (2007).

[5] R.N. Mohapatra and P.B. Pal, Massive Neutrinos in Physics and Astrophysics, third edition, World Scientific, (2004).

[6] B. Kayser, Neutrino Mass, Mixing and Flavor Change, in Neutrino Mass, Vol. 190, G. Höhler et al. eds. Springer, Berlin, Germany (2003) pp. 1-24.

[7] S.M. Bilenky and S.T. Petcov, Massive Neutrinos and Neutrino Oscillations, Rev. Mod. Phys. 59 (1987) 671 [INSPIRE].

[8] S.M. Bilenky, S.T. Petcov and B. Pontecorvo, Lepton Mixing, $\mu \rightarrow e+\gamma$ Decay and Neutrino Oscillations, Phys. Lett. B 67 (1977) 309 [InSPIRE].

[9] B.W. Lee and R.E. Shrock, Natural suppression of symmetry violation in gauge theories: Muon- and electron-lepton number nonconservation, Phys. Rev. D 16 (1977) 1444 [INSPIRE].

[10] W.J. Marciano and A.I. Sanda, Exotic decays of the muon and heavy leptons in gauge theories, Phys. Lett. B 67 (1977) 303 [INSPIRE].

[11] S.T. Petcov, The processes $\mu \rightarrow e+\gamma, \mu \rightarrow e+e+\bar{e}, \nu^{\prime} \rightarrow \nu+\gamma$ in the Weinberg-Salam model with neutrino mixing, Sov. J. Nucl. Phys. 25 (1977) 340 [INSPIRE].

[12] R.E. Shrock, Electromagnetic properties and decays of Dirac and Majorana neutrinos in a general class of gauge theories, Nucl. Phys. B 206 (1982) 359 [INSPIRE].

[13] P.B. Pal and L. Wolfenstein, Radiative decays of massive neutrinos, Phys. Rev. D 25 (1982) 766 [INSPIRE].

[14] S.L. Glashow, J. Iliopoulos and L. Maiani, Weak interactions with lepton-hadron symmetry, Phys. Rev. D 2 (1970) 1285 [InSPIRE].

[15] J.F. Nieves, Electromagnetic properties of Majorana neutrinos, Phys. Rev. D 26 (1982) 3152 [INSPIRE].

[16] R.E. Shrock, Electromagnetic properties and decays of Dirac and Majorana neutrinos in a general class of gauge theories, Nucl. Phys. B 206 (1982) 359 [InSPIRE].

[17] M.S. Dvornikov and A.I. Studenikin, Electromagnetic form-factors of a massive neutrino, J. Exp. Theor. Phys. 99 (2004) 254 [hep-ph/0411085] [INSPIRE].

[18] M. Dvornikov and A. Studenikin, Electric charge and magnetic moment of massive neutrino, Phys. Rev. D 69 (2004) 073001 [hep-ph/0305206] [INSPIRE]. 
[19] C. Giunti and A. Studenikin, Neutrino electromagnetic properties, Phys. Atom. Nucl. 72 (2009) 2089 [arXiv: 0812.3646] [INSPIRE].

[20] C. Broggini, C. Giunti and A. Studenikin, Electromagnetic Properties of Neutrinos, Adv. High Energy Phys. 2012 (2012) 459526 [arXiv: 1207.3980] [INSPIRE].

[21] A.A. Gvozdev, N.V. Mikheev and L.A. Vasilevskaya, The magnetic catalysis of the radiative decay of a massive neutrino in the standard model with lepton mixing, Phys. Lett. B 289 (1992) 103 [INSPIRE].

[22] V.C. Zhukovsky, P.A. Eminov and A.E. Grigoruk, Radiative decay of a massive neutrino in the Weinberg-Salam model with mixing in a constant uniform magnetic field, Mod. Phys. Lett. A 11 (1996) 3119 [INSPIRE].

[23] M. Kachelriess and G. Wunner, Radiative neutrino decays in very strong magnetic fields, Phys. Lett. B 390 (1997) 263 [hep-ph/9610439] [INSPIRE].

[24] A.A. Gvozdev, N.V. Mikheev and L.A. Vasilevskaya, Resonance neutrino bremsstrahlung $\nu \rightarrow \nu \gamma$ in a strong magnetic field, Phys. Lett. B 410 (1997) 211 [hep-ph/9702285] [INSPIRE].

[25] A.N. Ioannisian and G.G. Raffelt, Cherenkov radiation by massless neutrinos in a magnetic field, Phys. Rev. D 55 (1997) 7038 [hep-ph/9612285] [INSPIRE].

[26] R.A. Anikin and N.V. Mikheev, Radiative neutrino decay in a strong magnetic field, Phys. Atom. Nucl. 76 (2013) 1541 [INSPIRE].

[27] A.I. Ternov and P.A. Eminov, Neutrino radiative decay in external field and medium, Phys. Part. Nucl. 45 (2014) 397 [InSPIRE].

[28] A.A. Gvozdev, N.V. Mikheev and L.A. Vasilevskaya, The radiative decay of a high-energy neutrino in the Coulomb field of a nucleus, Phys. Lett. B 323 (1994) 179 [hep-ph/9404289] [INSPIRE].

[29] E.A. Choban and A.N. Ivanov, The Production of Lepton Pairs by High-energy Neutrinos in the Field of a Strong Electromagnetic Wave, Sov. Phys. JETP 29 (1969) 109.

[30] A.V. Borisov, V.C. Zhukovsky and A.I. Ternov, Electron positron pair production by a neutrino in an external electromagnetic field, Phys. Lett. B 318 (1993) 489 [INSPIRE].

[31] A.V. Kuznetsov and N.V. Mikheev, The neutrino energy and momentum loss through the process $\nu \rightarrow \nu e^{-} e^{+}$in a strong magnetic field, Phys. Lett. B 394 (1997) 123 [hep-ph/9612312] [INSPIRE].

[32] A.V. Kuznetsov, N.V. Mikheev and D.A. Rumyantsev, Lepton pair production by high-energy neutrino in an external electromagnetic field, Mod. Phys. Lett. A 15 (2000) 573 [hep-ph/0003216] [INSPIRE].

[33] A.V. Kuznetsov, N.V. Mikheev and D.A. Rumyantsev, Lepton-pair production by a neutrino in an external electromagnetic field, Phys. Atom. Nucl. 65 (2002) 277 [InSPIRE].

[34] T.M. Tinsley, Pair production with neutrinos and high-intensity laser fields, Phys. Rev. D 71 (2005) 073010 [hep-ph/0412014] [INSPIRE].

[35] D.A. Dicus, W.W. Repko and T.M. Tinsley, Pair production with neutrinos in an intense background magnetic field, Phys. Rev. D 76 (2007) 025005 [Erratum ibid. D 76 (2007) 089903] [arXiv:0704.1695] [INSPIRE].

[36] A.V. Kuznetsov, N.V. Mikheev, G.G. Raffelt and L.A. Vassilevskaya, Neutrino dispersion in external magnetic fields, Phys. Rev. D 73 (2006) 023001 [hep-ph/0505092] [INSPIRE]. 
[37] A. Erdas, Neutrino self-energy in external magnetic field, Phys. Rev. D 80 (2009) 113004 [arXiv: 0908.4297] [INSPIRE].

[38] A.A. Dobrynina, N.V. Mikheev and E.N. Narynskaya, Neutrino self-energy operator and neutrino magnetic moment, Phys. Atom. Nucl. 76 (2013) 1352 [INSPIRE].

[39] A.M. Egorov, A.E. Lobanov and A.I. Studenikin, Neutrino oscillations in electromagnetic fields, Phys. Lett. B 491 (2000) 137 [hep-ph/9910476] [INSPIRE].

[40] M.S. Dvornikov and A.I. Studenikin, Neutrino oscillations in the field of a linearly polarized electromagnetic wave, Phys. Atom. Nucl. 64 (2001) 1624 [INSPIRE].

[41] A.E. Lobanov and A.I. Studenikin, Neutrino oscillations in moving and polarized matter under the influence of electromagnetic fields, Phys. Lett. B 515 (2001) 94 [hep-ph/0106101] [INSPIRE].

[42] M.S. Dvornikov and A.I. Studenikin, Parametric resonance in neutrino oscillations in periodically varying electromagnetic fields, Phys. Atom. Nucl. 67 (2004) 719 [INSPIRE].

[43] A. Lobanov and A. Studenikin, Spin light of neutrino in matter and electromagnetic fields, Phys. Lett. B 564 (2003) 27 [hep-ph/0212393] [InSPIRE].

[44] A. Studenikin and A. Ternov, Neutrino quantum states and spin light in matter, Phys. Lett. B 608 (2005) 107 [hep-ph/0412408] [INSPIRE].

[45] V.V. Skobelev, The $\gamma \rightarrow \nu \bar{\nu}$ and $\nu \rightarrow \gamma \nu$ reactions in strong magnetic fields, Sov. Phys. JETP 44 (1976) 660.

[46] L.L. DeRaad K.A. Milton and N.D. Hari Dass, Photon Decay Into Neutrinos in a Strong Magnetic Field, Phys. Rev. D 14 (1976) 3326 [INSPIRE].

[47] A.V. Kuznetsov, N.V. Mikheev and L.A. Vassilevskaya, Photon splitting $\gamma \rightarrow \nu \bar{\nu}$ in an external magnetic field, Phys. Lett. B 427 (1998) 105 [hep-ph/9712289] [INSPIRE].

[48] R. Shaisultanov, Photon neutrino interactions in magnetic field, Phys. Rev. Lett. 80 (1998) 1586 [hep-ph/9709420] [INSPIRE].

[49] M.V. Chistyakov and N.V. Mikheev, Photon neutrino interactions in strong magnetic field, Mod. Phys. Lett. A 17 (2002) 2553 [hep-ph/0211146] [INSPIRE].

[50] A.V. Kuznetsov, N.V. Mikheev and D.A. Rumyantsev, Process $\gamma \gamma \rightarrow \nu \bar{\nu}$ in a strong magnetic field, Phys. Atom. Nucl. 66 (2003) 294 [inSPIRE].

[51] A.I. Titov, B. Kämpfer, H. Takabe and A. Hosaka, Neutrino pair emission off electrons in a strong electromagnetic wave field, Phys. Rev. D 83 (2011) 053008 [arXiv:1011.4860] [INSPIRE].

[52] A. Kuznetsov and N. Mikheev, Electroweak Processes in External Active Media, Springer, (2013).

[53] A. Kuznetsov and N. Mikheev, Electroweak Processes in External Electromagnetic Fields, Springer, (2003).

[54] S. Meuren, K.Z. Hatsagortsyan, C.H. Keitel and A. Di Piazza, Polarization operator approach to pair creation in short laser pulses, Phys. Rev. D 91 (2015) 013009 [arXiv: 1406.7235] [INSPIRE].

[55] A.A. Gvozdev, N.V. Mikheev and L.A. Vasilevskaya, The radiative decay $\nu_{i} \rightarrow \nu_{j} \gamma(i \neq j)$ of a massive neutrino in the field of an intensive electromagnetic wave, Phys. Lett. B 313 (1993) 161 [INSPIRE]. 
[56] A.A. Gvozdev, N.V. Mikheev and L.A. Vasilevskaya, Electromagnetic catalysis of the radiative transitions of $\nu_{i} \rightarrow \nu_{j} \gamma$ type in the field of an intense monochromatic wave, Phys. Lett. B 321 (1994) 108 [hep-ph/9404290] [INSPIRE].

[57] A.A. Gvozdev, N.V. Mikheev and L.A. Vasilevskaya, The radiative decay of the massive neutrino in the external electromagnetic fields, Phys. Rev. D 54 (1996) 5674 [hep-ph/9610219] [INSPIRE].

[58] V.V. Skobelev, Interaction between a massive neutrino and a plane wave field, Sov. Phys. JETP 73 (1991) 40 [INSPIRE].

[59] R. Mohammadi and S.-S. Xue, Laser photons acquire circular polarization by interacting with a Dirac or Majorana neutrino beam, Phys. Lett. B 731 (2014) 272 [arXiv:1312.3862] [INSPIRE].

[60] P. Bakhti, R. Mohammadi and S.-S. Xue, Using strong intense lasers to probe sterile neutrinos, arXiv: 1403.7327 [INSPIRE].

[61] A. Di Piazza, C. Müller, K.Z. Hatsagortsyan and C.H. Keitel, Extremely high-intensity laser interactions with fundamental quantum systems, Rev. Mod. Phys. 84 (2012) 1177 [arXiv:1111.3886] [INSPIRE].

[62] W. Dittrich and H. Gies, Probing the Quantum Vacuum, Springer, Berlin, Germany (2000).

[63] E.S. Fradkin, D.M. Gitman and S.M. Shvartsman, Quantum electrodynamics: with unstable vacuum, Springer-Verlag, (1991).

[64] V.I. Ritus, Quantum effects of the interaction of elementary particles with an intense electromagnetic field, J. Sov. Laser Res. 6 (1985) 497.

[65] H. Mitter, Quantum Electrodynamics in Laser Fields, Acta Phys. Austriaca Suppl. XIV (1975) 397.

[66] D.M. Wolkow, Über eine Klasse von Lösungen der Diracschen Gleichung, Z. Phys. 94 (1935) 250 [INSPIRE].

[67] L.D. Landau and E.M. Lifshitz, Quantum Electrodynamics, Butterworth-Heinemann, (1982).

[68] S. Meuren, C.H. Keitel and A. Di Piazza, Polarization operator for plane-wave background fields, Phys. Rev. D 88 (2013) 013007 [arXiv:1304.7672] [INSPIRE].

[69] D.V. Galtsov and N.S. Nikitina, Photoneutrino Processes in a Strong Field, Sov. Phys. JETP 35 (1972) 1047.

[70] M.Y. Borovkov, A.V. Kuznetsov and N.V. Mikheev, One loop amplitude of the transition $j \rightarrow f \bar{f} \rightarrow j^{\prime}$ in an external electromagnetic field, Phys. Atom. Nucl. 62 (1999) 1601 [INSPIRE].

[71] J. Schwinger, On gauge invariance and vacuum polarization, Phys. Rev. 82 (1951) 664 [INSPIRE].

[72] W. Heisenberg and H. Euler, Consequences of Dirac's theory of positrons, Z. Phys. 98 (1936) 714 [physics/0605038] [InSPIRE].

[73] F. Sauter, Über das Verhalten eines Elektrons im homogenen elektrischen Feld nach der relativistischen Theorie Diracs, Z. Phys. 69 (1931) 742 [INSPIRE].

[74] D.A. Dicus, A. Farzinnia, W.W. Repko and T.M. Tinsley, Muon decay in a laser field, Phys. Rev. D 79 (2009) 013004 [arXiv:0809.2367] [INSPIRE]. 
[75] A. Farzinnia, D.A. Dicus, W.W. Repko and T.M. Tinsley, Muon decay in a linearly polarized laser field, Phys. Rev. D 80 (2009) 073004 [arXiv:0907.1052] [INSPIRE].

[76] International scoping study of a future Neutrino Factory and super-beam facility, http://www.hep.ph.ic.ac.uk/iss/.

[77] The International Design Study for the Neutrino Factory, http://www.ids-nf.org/.

[78] Muon Accelerator Program (MAP), http://map.fnal.gov/.

[79] MAP, MICE collaboration, D.M. Kaplan, Muon Colliders and Neutrino Factories, arXiv: 1412.3487 [INSPIRE].

[80] M. Bogomilov et al., Neutrino factory, Phys. Rev. ST Accel. Beams 17 (2014) 121002 [INSPIRE].

[81] S. Geer, Muon colliders and neutrino factories, arXiv:1202.2140 [INSPIRE].

[82] ISS Accelerator Working Group collaboration, M. Apollonio et al., Accelerator design concept for future neutrino facilities, 2009 JINST 4 P07001 [arXiv:0802.4023] [INSPIRE].

[83] A. Bandyopadhyay et al., Physics at a future Neutrino Factory and super-beam facility, Rept. Prog. Phys. 72 (2009) 106201.

[84] J.S. Berg et al., A cost-effective design for a neutrino factory, Phys. Rev. ST Accel. Beams 9 (2006) 011001 [physics/0511092] [INSPIRE].

[85] S. Geer, Neutrino beams from muon storage rings: Characteristics and physics potential, Phys. Rev. D 57 (1998) 6989 [hep-ph/9712290] [INSPIRE].

[86] Extreme Light Infrastructure (ELI), http://www.eli-laser.eu/.

[87] Central Laser Facility (CLF), http://www.stfc.ac.uk/clf/.

[88] Exawatt Center for Extreme Light Studies (XCELS), http://www.xcels.iapras.ru/.

[89] V. Yanovsky et al., Ultra-high intensity-300-TW laser at $0.1 \mathrm{~Hz}$ repetition rate, Opt. Express 16 (2008) 2109.

[90] H. Gies, F. Karbstein and R. Shaisultanov, Laser photon merging in an electromagnetic field inhomogeneity, Phys. Rev. D 90 (2014) 033007 [arXiv: 1406.2972] [INSPIRE].

[91] V. Dinu, T. Heinzl, A. Ilderton, M. Marklund and G. Torgrimsson, Photon polarization in light-by-light scattering: Finite size effects, Phys. Rev. D 90 (2014) 045025 [arXiv: 1405.7291] [INSPIRE].

[92] V. Dinu, T. Heinzl, A. Ilderton, M. Marklund and G. Torgrimsson, Vacuum refractive indices and helicity flip in strong-field QED, Phys. Rev. D 89 (2014) 125003 [arXiv: 1312.6419] [INSPIRE].

[93] V.N. Baier, A.I. Mil'shtein and V.M. Strakhovenko, Interaction between a photon and an intense electromagnetic wave, Sov. Phys. JETP 42 (1975) 961.

[94] W. Becker and H. Mitter, Vacuum polarization in laser fields, J. Phys. A 8 (1975) 1638.

[95] H. Gies and R. Shaisultanov, On the axial current in an electromagnetic field and low-energy neutrino-photon interactions, Phys. Rev. D 62 (2000) 073003 [hep-ph/0003144] [INSPIRE].

[96] H. Gies and R. Shaisultanov, Neutrino interactions with a weak slowly varying electromagnetic field, Phys. Lett. B 480 (2000) 129 [hep-ph/0009342] [INSPIRE].

[97] R. Shaisultanov, The neutrino neutrino gamma amplitude in an external homogeneous electromagnetic field, Phys. Rev. D 62 (2000) 113005 [hep-th/0002079] [INSPIRE]. 
[98] C. Schubert, Vacuum polarization tensors in constant electromagnetic fields. Part 1., Nucl. Phys. B 585 (2000) 407 [hep-ph/0001288] [INSPIRE].

[99] C. Schubert, Vacuum polarization tensors in constant electromagnetic fields. Part 2., Nucl. Phys. B 585 (2000) 429 [hep-ph/0002276] [INSPIRE].

[100] H. Gies and C. Schubert, Vacuum polarization tensors in constant electromagnetic fields. Part 3., Nucl. Phys. B 609 (2001) 313 [hep-ph/0104077] [InSPIRE].

[101] K. Bhattacharya and A.K. Ganguly, The axialvector vector amplitude and neutrino effective charge in a magnetized medium, Phys. Rev. D 68 (2003) 053011 [hep-ph/0308063] [INSPIRE].

[102] J.F. Nieves, The electromagnetic vertex of neutrinos in an electron background and a magnetic field, Phys. Rev. D 68 (2003) 113003 [hep-ph/0309240] [INSPIRE].

[103] S.L. Adler, Axial vector vertex in spinor electrodynamics, Phys. Rev. 177 (1969) 2426 [INSPIRE].

[104] J.S. Bell and R. Jackiw, A PCAC puzzle: $\pi 0 \rightarrow \gamma \gamma$ in the $\sigma$-model, Nuovo Cim. A 60 (1969) 47 [INSPIRE].

[105] D. Bardin and G. Passarino, The Standard Model in the Making: Precision Study of the Electroweak Interactions, Clarendon Press, Oxford U.K. (1999).

[106] J.F. Donoghue, E. Golowich and B.R. Holstein, Dynamics of the Standard Model, Cambridge University Press, (1994).

[107] S. Pokorski, Gauge Field Theories, second edition, Cambridge University Press, (2000).

[108] T. Cheng and L. Li, Gauge theory of elementary particle physics, Clarendon Press, (1982).

[109] K.I. Aoki, Z. Hioki, M. Konuma, R. Kawabe and T. Muta, Electroweak Theory. Framework of On-Shell Renormalization and Study of Higher Order Effects, Prog. Theor. Phys. Suppl. 73 (1982) 1 [INSPIRE].

[110] M. Böhm, H. Spiesberger and W. Hollik, On the One Loop Renormalization of the Electroweak Standard Model and Its Application to Leptonic Processes, Fortsch. Phys. 34 (1986) 687 [INSPIRE].

[111] W.F.L. Hollik, Radiative Corrections in the Standard Model and their Role for Precision Tests of the Electroweak Theory, Fortsch. Phys. 38 (1990) 165 [InSPIRE].

[112] A. Denner, Techniques for calculation of electroweak radiative corrections at the one loop level and results for W physics at LEP-200, Fortsch. Phys. 41 (1993) 307 [arXiv:0709.1075] [INSPIRE].

[113] B. Kayser, On the Quantum Mechanics of Neutrino Oscillation, Phys. Rev. D 24 (1981) 110 [INSPIRE].

[114] B. Pontecorvo, Mesonium and anti-mesonium, Sov. Phys. JETP 6 (1957) 429 [INSPIRE].

[115] B. Pontecorvo, Inverse beta processes and nonconservation of lepton charge, Sov. Phys. JETP 7 (1958) 172 [INSPIRE].

[116] Z. Maki, M. Nakagawa and S. Sakata, Remarks on the unified model of elementary particles, Prog. Theor. Phys. 28 (1962) 870 [INSPIRE].

[117] E. Fermi, Trends to a Theory of beta Radiation. (In Italian), Nuovo Cim. 11 (1934) 1 [INSPIRE]. 
[118] E. Fermi, An attempt of a theory of beta radiation. 1., Z. Phys. 88 (1934) 161 [InSPIRE].

[119] P.A.M. Dirac, Forms of Relativistic Dynamics, Rev. Mod. Phys. 21 (1949) 392 [InSPIRE].

[120] R.A. Neville and F. Rohrlich, Quantum electrodynamics on null planes and applications to lasers, Phys. Rev. D 3 (1971) 1692 [InSPIRE].

[121] S. Meuren, C.H. Keitel and A. Di Piazza, Semiclassical description of nonlinear electron-positron photoproduction in strong laser fields, arXiv:1503.03271 [INSPIRE].

[122] R. Battesti and C. Rizzo, Magnetic and electric properties of a quantum vacuum, Rep. Prog. Phys. 76 (2013) 016401.

[123] F. Ehlotzky, K. Krajewska and J.Z. Kamiński, Fundamental processes of quantum electrodynamics in laser fields of relativistic power, Rep. Prog. Phys. 72 (2009) 046401.

[124] G.A. Mourou, T. Tajima and S.V. Bulanov, Optics in the relativistic regime, Rev. Mod. Phys. 78 (2006) 309 [INSPIRE].

[125] M. Marklund and P.K. Shukla, Nonlinear collective effects in photon-photon and photon-plasma interactions, Rev. Mod. Phys. 78 (2006) 591 [hep-ph/0602123] [InSPIRE].

[126] D.J. Gross and R. Jackiw, Effect of anomalies on quasirenormalizable theories, Phys. Rev. D 6 (1972) 477 [INSPIRE].

[127] K. Nishijima, Generalized Furry's Theorem for Closed Loops, Progr. Theor. Phys. 6 (1951) 614.

[128] R. Jackiw and K. Johnson, Anomalies of the axial vector current, Phys. Rev. 182 (1969) 1459 [INSPIRE].

[129] S.L. Adler and W.A. Bardeen, Absence of higher order corrections in the anomalous axial vector divergence equation, Phys. Rev. 182 (1969) 1517 [INSPIRE].

[130] W.A. Bardeen, Anomalous Ward identities in spinor field theories, Phys. Rev. 184 (1969) 1848 [INSPIRE].

[131] K. Fujikawa, Path Integral Measure for Gauge Invariant Fermion Theories, Phys. Rev. Lett. 42 (1979) 1195 [INSPIRE].

[132] M.E. Peskin and D.V. Schroeder, An Introduction to Quantum Field Theory, Perseus Books, (1995).

[133] S. Weinberg, The Quantum Theory of Fields II, Cambridge University Press, (1996).

[134] V.I. Ritus, Radiative corrections in quantum electrodynamics with intense field and their analytical properties, Annals Phys. 69 (1972) 555 [INSPIRE].

[135] F.W.J. Olver, D.W. Lozier, R.F. Boisvert and C.W. Clark, NIST Handbook of Mathematical Functions, Cambridge University Press, (2010).

[136] A.I. Nikishov and V.I. Ritus, Quantum Processes in the Field of a Plane Electromagnetic Wave and in a Constant Field 1, Sov. Phys. JETP 19 (1964) 529 [INSPIRE].

[137] S. Meuren, K.Z. Hatsagortsyan, C.H. Keitel and A. Di Piazza, High-Energy Recollision Processes of Laser-Generated Electron-Positron Pairs, Phys. Rev. Lett. 114 (2015) 143201 [arXiv: 1407.0188] [INSPIRE].

[138] E. Leader, Spin in Particle Physics, Cambridge University Press, (2001). 